\title{
Incorporation of Stem Cell-Derived Astrocytes into Neuronal Organoids to Allow Neuro-Glial Interactions in Toxicological Studies
}

\author{
Markus Brüll1\#, Anna-Sophie Spreng1,2\#, Simon Gutbier ${ }^{3}$, Dominik Loser 4 , Alice Krebs 1,2, Marvin Reich 1,3, \\ Udo Kraushaar ${ }^{4}$, Markus Britschgi ${ }^{3}$, Christoph Patsch ${ }^{3}$ and Marcel Leist ${ }^{1,2}$ \\ ${ }^{1}$ In vitro Toxicology and Biomedicine, Dept inaugurated by the Doerenkamp-Zbinden Foundation, University of Konstanz, Konstanz, Germany; \\ ${ }^{2}$ Konstanz Research School Chemical Biology (KoRS-CB), University of Konstanz, Konstanz, Germany; ${ }^{3}$ Roche Pharma Research and Early \\ Development, Roche Innovation Center Basel, Basel, Switzerland; ${ }^{4}$ NMI Natural and Medical Sciences Institute at the University of Tuebingen, \\ Reutlingen, Germany
}

\begin{abstract}
Human cell-based neural organoids are increasingly being used for investigations of neurotoxicity and to study the pathophysiology of neurodegenerative diseases. Here, we present a fast and robust method to generate 3D cultured human dopaminergic neurons (LUHMES) for toxicity testing and long-term culture. Moreover, a plating step was introduced to allow generation of neurite networks with a defined 2D orientation and several $\mathrm{mm}$ length while all cell bodies (somata) remained in a 3D, dome-like structure. These cultures, named 2.5D (for 2.5 dimensional), offer new approaches to quantify toxicant effects on organoids by standard technology and in high throughput. For instance, the system reacted to the parkinsonian model toxicants MPP+, rotenone and MG-132, and to the ferroptosis-inducer erastin. Moreover, stable incorporation of human stem cell-derived astrocytes or microglia was possible. Addition of astrocytes stabilized the post mitotic state of the LUHMES neurons and thereby allowed formation of a stable microphysiological system. Neuroprotection against the proteasome inhibitor MG-132 and the ferroptosis-inducer erastin was mediated by such glia, exemplifying the crucial protective role of astrocytes in neurodegeneration. The modularity of the system was further employed to incorporate microglia together with astrocytes into the organoids. Such ratio-defined, three cell type-based organoids will allow new approaches to study human pathophysiology and toxicology of the nervous system.
\end{abstract}

\section{Introduction}

The transition of neuronal cultures from a conventional monolayer (2D) approach towards the generation of 3D organoids offers new opportunities, but also entails various complications. Besides increased complexity in handling procedures, the transition requires the ability to co-culture neurons with other relevant cells of the nervous system. Moreover, setting up quantitative test methods using organoid systems requires new approaches to the assessment of physiological parameters, the modelling of pathophysiological processes, or the assessment of reactions to toxicants. To allow work on so many different aspects of cell culture and to identify the most efficient steps for further develop- ment, sequential probing and optimization of the above features is a useful strategy. Our study addresses several of the above issues and exemplifies some of the respective solutions.

The human brain differs vastly from the commonly used rodent brain models in terms of developmental time scale (Semple et al., 2013; Zimmer et al., 2011), structure (Florio and Huttner, 2014), and cellular composition/function (Zhang et al., 2016; Nedergaard et al., 2003; Polioudakis et al., 2019). Considering these inter-species differences, it is not surprising that established animal models often lack predictivity for humans (Leist and Hartung, 2013; Hartung and Leist, 2008; van der Worp et al., 2010; Leist et al., 2008). Human cell-based in vitro systems have therefore been used increasingly in neurotoxicological research

\# contributed equally

Received November 11, 2019; Accepted March 4, 2020; Epub March 7, 2020; ๔ The Authors, 2020.

ALTEX 37(3), 409-428. doi:10.14573/altex.1911111

Correspondence: Marcel Leist, PhD

In vitro Toxicology and Biomedicine

Dept inaugurated by the Doerenkamp-Zbinden Foundation

University of Konstanz

Universitätsstr. 10, 78464 Konstanz, Germany

(marcel.leist@uni-konstanz.de)
This is an Open Access article distributed under the terms of the Creative Commons Attribution 4.0 International license (http://creativecommons.org/licenses/by/4.0/), which permits unrestricted use, distribution and reproduction in any medium, provided the original work is appropriately cited. 
(Barbosa et al., 2015; Pamies and Hartung, 2017; Schmidt et al., 2017; Schildknecht et al., 2011; Stiegler et al., 2011). Breakthroughs in induced pluripotent stem cell (iPSC) technology have supported this trend (Takahashi et al., 2007; Shi et al., 2017; Tukker et al., 2018).

Traditional two-dimensional (2D) cell culturing also fails to replicate some crucial features of in vivo physiology (Marx et al., 2016). This includes cell maturation, cell-cell and cell-matrix interactions, spatio-temporal concentration gradients, and structural complexity (Pampaloni et al., 2007; Ravi et al., 2015; Sloan et al., 2017; Gieseck III et al., 2014; Marton and Pasca, 2016). To bridge this gap between in vivo and in vitro, multiple three-dimensional (3D) brain organoid models based on human cells have been developed. For instance, the Knoblich group and others used 3D-differentiation of pluripotent stem cells (PSC) to closely recapitulate human brain development and to generate cerebral organoids with sophisticated self-organization (Lancaster et al., 2013, 2017; Lancaster and Knoblich, 2014; Pasca et al., 2015; Trujillo et al., 2019; Yoon et al., 2019; Amiri et al., 2018). Similar approaches were used to generate cortical (Kadoshima et al., 2013), midbrain (Jo et al., 2016; Smits et al., 2019), forebrain (Qian et al., 2016), retina (Eiraku et al., 2011; Kim et al., 2019) or cerebellar organoids (Muguruma et al., 2015). Others generated neural 3D cultures from neural precursor cells (NPC) (Pamies et al., 2017) or neuroepithelial stem cells (NESC) (Monzel et al., 2017) instead of PSC. Typically, stem cell-derived brain organoids contain a large variety of neuronal subtypes, astrocytes (Dezonne et al., 2017, Yakoub, 2019), myelinating oligodendrocytes (Pamies et al., 2017; Monzel et al., 2017) and, in some cases, even microglia (Ormel et al., 2018).

While brain organoids differentiated from stem or progenitor cells closely mimic in vivo development (Camp et al., 2015) and physiology (Quadrato et al., 2017), their generation follows long and intricate protocols. These systems also do not allow control over the exact final cellular composition and maturity. Achieving reproducibility of the approach requires large efforts (Kelava and Lancaster, 2016; Pasca et al., 2015; Yoon et al., 2019).

Simplified approaches to generating neuronal organoids utilize the 3D culture of neuronal cell lines like NT2 (Serra et al., 2007; Simão et al., 2016) or LUHMES cells (Harris et al., 2017, 2018; Smirnova et al., 2016). The latter is a conditionally immortalized human neuronal progenitor cell line that can be differentiated into mature dopaminergic neurons within six days (Lotharius et al., 2005; Scholz et al., 2011). LUHMES cells have been extensively characterized (Gutbier et al., 2018a; Delp et al., 2018a; Schildknecht et al., 2013b) and have been widely used to model neurodegeneration (Zhang et al., 2014; Efremova et al., 2017; Scholz et al., 2018; Gutbier et al., 2018b) and for neurotoxicity testing (Delp et al., 2019, 2018b; Krug et al., 2013, 2014; Tong et al., 2017; Singh et al., 2018; Skirzewski et al., 2018; Witt et al., 2017). Spheroid culture of LUHMES allows extended ex- perimental time frames (Harris et al., 2018; Smirnova et al., 2016) and increases the complexity and in vivo relevance of the system. Compared to stem cell-derived spheroids, 3D cultures of LUHMES do not contain other neural cell types, e.g., astrocytes or microglia.

However, the presence of glia, i.e., microglia and astrocytes, in brain organoids may be the key to approaching physiological functions of neurons further. Microglia are important for removal of cell debris, for pathogen recognition, and as generators of oxidative stress (Schildknecht et al., 2013a; Hirt et al., 2000; Falsig et al., 2008). Astrocytes play a crucial role in brain metabolism, e.g., by recycling neurotransmitters (Sidoryk-Wegrzynowicz et al., 2011) or as a source of cellular thiols (Wang and Cynader, 2000; Gutbier et al., 2018b). They also control synapse formation and elimination (Chung et al., 2015; Eroglu and Barres, 2010; Wilton et al., 2019; Jay et al., 2019; Klapper et al., 2019). Moreover, astrocytes may play important roles in toxicology: On the one hand, astrocytes have been shown to be neuroprotective (Bélanger and Magistretti, 2009; Chen et al., 2009; Gutbier et al., 2018b; Rathinam et al., 2012; Stary et al., 2015; Bylicky et al., 2018), while on the other hand, they can trigger neurodegeneration upon inflammatory activation (Li et al., 2019; Efremova et al., 2017; Falsig et al., 2004) or by metabolizing pro-toxicants (Schildknecht et al., 2015). They are therefore of crucial importance for modelling of the brain environment and in-vivo-like responses to toxicants.

Co-culture of differentiated neurons and astrocytes was used in multiple 2D models to investigate neuro-glial interactions with precise control over cellular composition (Jones et al., 2012; Efremova et al., 2015, 2017; Gutbier et al., 2018b; Roque and Costa, 2017). This was also explored in organoid cultures (Krencik et al., 2017). Spheroid co-culture of LUHMES and glia therefore offers a systematic approach to emulating the cellular complexity of the human brain while retaining control of the cellular composition and being able to produce organoids rapidly.

Many of the established organoid culture systems use spinning bioreactors or shakers (Harris et al., 2017; Lancaster and Knoblich, 2014; Pamies et al., 2017; Honegger and Richelson, 1977, 1979). However, it is unclear whether such spontaneous self-organization methods allow the generation of co-culture organoids with pre-defined cell ratios. Another approach utilizes hanging drops to generate neuronal spheroids. This approach allows precise control over cellular composition (Nzou et al., 2018), but it involves cumbersome handling. Spheroid generation from defined numbers of cells is also possible by using round bottom ultra-low attachment (ULA) plates (Howes et al., 2014). Definition of exact ratios of all populations is of interest, not only for co-cultures (e.g., systematic variation of the neuron-glia ratio), but it is also important, e.g., if fluorescent or reporter cells are to be added to neuronal mono-cultures (Schildknecht et al., 2013b; Scholz et al., 2011; Krug et al., 2014).

\section{Abbreviations}

2D, two-dimensional; 3D, three-dimensional; DAT, dopamine transporter; DMEM, Dulbecco's modified Eagle's medium; EB, embryoid body; EdU, 5-ethynyl-2'-deoxyuridine; FBS, fetal bovine serum; FGF, fibroblast growth factor; GFAP, glial fibrillary acidic protein; iPSC, induced pluripotent stem cells; LUHMES, Lund human mesencephalic; MEA, micro-electrode array; MGV, mean grey value; MPP+,1-methyl-4-phenylpyridinium; NF200, neurofilament 200; PBS, phosphate buffered serine; PI, propidium iodide; PLO, poly-L-ornithine; PSC, pluripotent stem cells; PSD95, post-synaptic density protein 95; RFP, red fluorescent protein; RT, room temperature; SYP, synaptophysin; ULA, ultra-low attachment 
In this study, we developed a ULA plate-based system to generate LUHMES organoids that can be handled individually. By co-culturing LUHMES with mature human stem-cell derived astrocytes and microglia, we were able to reproducibly create more complex, post mitotic, brain-like organoids. This modular approach offers precise control over cellular composition, allows the investigation of neuron-glia interactions, and provides new opportunities for neurotoxicity testing.

\section{Materials and methods}

\subsection{Chemicals}

Erastin and MG-132 were purchased from Selleckchem, USA. Veratridine and $\alpha, \beta$-meATP were purchased from Tocris, UK. $\mathrm{MPP}^{+}$, rotenone, carbaryl, valinomycin, colchicine, GBR-12909, (-)-nicotine, Triton-X100, calcein-AM and D-sorbitol were all purchased from Sigma-Aldrich, USA. Urea was purchased from Roth, Germany.

\subsection{D LUHMES cell culture}

LUHMES cells were handled as previously described in detail (Scholz et al., 2011). Proliferating LUHMES cells were typically cultivated in PLO/fibronectin-coated T75 flasks (Sarstedt, Germany) in $10 \mathrm{~mL}$ proliferation medium. Proliferation medium consisted of advanced DMEM/F12 (Gibco, USA) supplemented with $2 \mathrm{mM}$ glutamine (Sigma-Aldrich, USA) 1x N2 supplement (Gibco, USA) and $40 \mu \mathrm{g} / \mathrm{mL}$ fibroblast growth factor (FGF) (R\&D Systems, USA). Cells were passaged into a new flask every 2-3 days when they reached approximately $80 \%$ confluence. To passage the cells, the medium was removed and the cells were washed with $10 \mathrm{~mL}$ phosphate buffered saline (PBS) (Merck Millipore, Germany). For detachment, cells were incubated for $3 \mathrm{~min}$ at $37^{\circ} \mathrm{C}$ in $2 \mathrm{~mL} 0.05 \%$ trypsin-ethylenediaminetetraacetic acid (EDTA) solution (Gibco, USA). The trypsinized cell suspension was added to $13 \mathrm{~mL}$ advanced DMEM/F12 without supplements. The cell suspension was centrifuged at $300 \mathrm{x} g$ for 5 min (1200 rpm, Heraeus Multifuge 1 S-R, ThermoScientific, USA) to wash out the trypsin. The supernatant was removed and the pellet was resuspended in $1 \mathrm{~mL}$ of advanced DMEM/F12. Cell number was determined by counting in a Neubauer counting chamber. Cells were seeded at a confluency of 30,000 cells/ $\mathrm{cm}^{2}$ for two days of proliferation; and at 15000 cells $/ \mathrm{cm}^{2}$ for three days of proliferation. Cells were cultivated at $37^{\circ} \mathrm{C}$ with $5 \% \mathrm{CO}_{2}$. To initiate differentiation, proliferating LUHMES cells were seeded at a confluency of 87,000 cells $/ \mathrm{cm}^{2}$ in differentiation medium (displayed as day 0/d0 in figures). LUHMES differentiation medium contained advanced DMEM-F12 supplemented with $2 \mathrm{mM}$ glutamine, 1x N2 supplement, $1 \mathrm{mM}$ dibutyrylcAMP (Sigma-Aldrich, USA), $2 \mathrm{ng} / \mathrm{mL}$ glial cell-derived neurotrophic factor (GDNF) (R\&D Systems, USA) and $2.25 \mu \mathrm{M}$ tetracycline (Sigma-Aldrich, USA). LUHMES cells were differentiated for two days before sphere generation.
Red fluorescent protein expressing LUHMES (RFP-LUHMES) were cultivated in the same way. They were produced as previously described by our group (Schildknecht et al., 2013b). Briefly, proliferating LUHMES cells were infected by lentiviruses containing a turbo-RFP gene under a cytomegalovirus (CMV)promotor. RFP-expressing LUHMES were selected by manually picking red fluorescent colonies under a fluorescent microscope. The colonies were propagated and the picking was repeated until all cells showed red fluorescence. The culture medium of all used cell lines was regularly checked for mycoplasma contamination by Venor ${ }^{\circledR}$ GeM Classic kit (Minerva Biolabs, Berlin, Germany). LUHMES cells working stock was validated by short tandem repeat profiling.

\subsection{Astro.4U cell culture}

Human iPSC-derived Astro.4U astrocytes were kindly provided by Ncardia, Germany, and used exactly as described previously (Gutbier et al., 2018b). Briefly, cryopreserved cells were thawed in $9 \mathrm{~mL}$ Neuro.4U basal medium (Astro.4U kit, Ncardia, Germany) according to the manufacturer's handling guide. They were centrifuged at $300 \mathrm{xg}$ for $5 \mathrm{~min}$. The pellet was resuspended in Astro.4U culture medium and seeded at a density of 40,000 cells $/ \mathrm{cm}^{2}$ on Matrigel (Corning, USA). For maintenance, medium was exchanged completely every three to four days. The astrocytes used in our experiments were comprehensively characterized: They expressed typical astroglial markers (glial fibrillary acidic protein (GFAP), aquaporin (AQP)4, monoamine oxidase B (Mao-B)) (Fig. S1A, ${ }^{1}$ ), were responsive to inflammatory stimuli, and were fully post mitotic (data not shown). When co-cultured with differentiated LUHMES cells, they changed shape from a triangular, flattened morphology to the typical starshaped morphology (Fig. S1B ${ }^{1}$ ). For use in co-culture organoids, Astro.4U were detached with Accutase ${ }^{\circledR}$ (Corning, USA) for $5 \mathrm{~min}$ at $37^{\circ} \mathrm{C}$. Cells were washed off with DMEM/F-12 and centrifuged for $5 \mathrm{~min}$ at $300 \mathrm{x} \mathrm{g}$ (1200 rpm, Heraeus Multifuge $1 \mathrm{~S}-\mathrm{R}$, ThermoScientific). Cells were resuspended in DMEM/ F-12, counted, and used for characterization or mixed with LUHMES (d2) cells in appropriate cell ratios. Cell characterization (morphology, marker expression, cytokine response) showed typical astrocyte characteristics, similar to Astro.4U.

\subsection{Astro.KN differentiation and culture}

Astro.KN were differentiated from the human stem cell line H9 (Thomson et al., 1998) according to Palm et al. (2015), until they reached the neural stem cell (NSC) state. At this state, NSC were cultivated for 49 days in NSC-medium consisting of DMEM/F12 (Gibco, USA) supplemented with N2 (0.5x; Gibco, USA), B27 with vitamin A (1x; Gibco, USA), L-glutamine (2 mM; SigmaAldrich, USA), FGF2 (20 ng/mL; R\&D, USA), epidermal growth factor (EGF) (20 ng/mL; R\&D, USA) and leukemia inhibitory factor (LIF)1010 (1.5 $\mu \mathrm{L} / \mathrm{mL}$; Merckmillipore, Germany). For further differentiation towards astrocytes, d49 NSC were seeded at a density of 50,000 cells $/ \mathrm{cm}^{2}$ on Matrigel-coated (1:20 di-

1 doi:10.14573/altex.1911111s1 
lution in DMEM/F-12) plates in NSC-medium. Astrocyte differentiation was induced after 2 days by changing the NSC-medium to astrocyte differentiation medium (1\% fetal bovine serum (FBS) in NSC medium without growth factors). Astrocytes were differentiated for at least 35 days with weekly passaging and medium change every other day. For passaging and incorporation into organoids, astrocytes were detached with Accutase ${ }^{\circledR}$ (Corning, USA) for $5 \mathrm{~min}$ at $37^{\circ} \mathrm{C}$, washed off with $\mathrm{DMEM} / \mathrm{F}-12$ and centrifuged at $500 \mathrm{x}$ g for $5 \mathrm{~min}$. Cells were resuspended in DMEM/F-12, then counted and mixed with LUHMES (d2) cells in appropriate cell ratios.

FBS has been used in many protocols to generate astrocytes from stem cells (Chandrasekaran et al., 2016) and also in the protocol adapted for our study. We have tried to replace FBS with human serum or human platelet lysate or with isolated morphogens such as BMP4. These attempts have been only partly successful. The differentiation with only $1 \%$ FBS still produces the best results in terms of efficacy and reproducibility, but we will keep looking for alternatives for future studies.

\subsection{Microglia culture}

For this protocol, an iPSC line (STBCi026-A, StemBANCC) was used that was engineered to stably express green fluorescent protein (GFP) under a constitutive promotor by lentiviral transfection. In order to obtain macrophage progenitors, iPS cells were differentiated as described in detail (van Wilgenburg et al., 2013; Haenseler et al., 2017). Briefly, iPS cells were detached by incubation with Accutase (Corning, USA) for $5 \mathrm{~min}$ at $37^{\circ} \mathrm{C}$. The cell suspension was then diluted with mTESR (StemCell Technologies, Canada) and centrifuged for 5 min at $300 \mathrm{x}$ g. Embryoid body (EB) formation was induced by addition of $4 \times 10^{6}$ cells per well in an Aggrewell 800 plate (StemCell Technologies, Canada) in mTESR containing $10 \mu \mathrm{M}$ Rock inhibitor Y27632 (Tocris, United Kingdom). For EB formation, cells were cultured for 4 days in Aggrewell plates, with a $66 \%$ medium change every $24 \mathrm{~h}$. For mesoderm induction, mTESR was supplemented with BMP4 (50 ng/mL; Peprotech, USA), VEGF (50 ng/mL; R\&D Systems, USA) and SCF (20 ng/mL; R\&D Systems, USA) at $24 \mathrm{~h}$ after replating. After 4 days of EB formation, EBs were dislodged and transferred to cell culture dishes in XVIVO 15 medium (Lonza, Switzerland) containing M-CSF (100 ng/mL; Miltenyi, Germany), IL-34 (25 ng/mL; Miltenyi, Germany), $\beta$-mercaptoethanol (50 $\mu \mathrm{M}$ Invitrogen, USA), Glutamaxx (2 mM, Invitrogen, USA) and PenStrep (Invitrogen, USA). Dishes were left untouched in the incubator for the following 7 days. The medium was partially (50\%) renewed at $\mathrm{d} 7$ and d14 after replating of the EBs. Once the "blood factory" stage was reached and the production of pre-macrophages was ongoing, medium was exchanged twice per week. Pre-macrophages were harvested from the supernatant by centrifugation. Pre-macrophages were differentiated toward microglia-like cells in XVIVO 15 medium supplemented with GlutaMAX ${ }^{\mathrm{TM}}$ (1x; Gibco, USA), Penicillin-Streptomycin (1x; Gibco, USA), IL-34 (100 ng/mL; R\&D Systems, USA) and GM-CSF (10 ng/ $\mathrm{mL}$; R\&D Systems, USA) for 7 days according to Haensler et al. (2017) (Fig. S2A ${ }^{1}$ ). The cells were characterized by immunostaining of microglia markers (Iba1, PU.1) and by their abili- ty to release cytokines upon stimulation with LPS (Fig. S2B, $\mathrm{C}^{1}$ ). Microglia-like cells were used for the incorporation in LUHMES organoids ( \pm astrocytes). For this, LUHMES differentiation medium was supplemented with Penicillin-Streptomycin, IL-34 (100 ng/mL) and GM-CSF (10 ng/mL).

\subsection{Coating of cell culture plates}

For maintenance culture and differentiation, LUHMES cells were seeded in T75 flasks (Sarstedt, Germany). To ensure homogenous growth and sufficient adhesion, the flasks were coated. Coating solution contained $43 \mu \mathrm{g} / \mathrm{mL}$ Poly-L-ornithine hydrobromide (PLO, Sigma-Aldrich, USA) and $1 \mu \mathrm{g} / \mathrm{mL}$ fibronectin (Sigma-Aldrich, USA) in milliQ $\mathrm{H}_{2} \mathrm{O}$. Flasks were incubated with $10 \mathrm{~mL}$ coating solution at $37^{\circ} \mathrm{C}$ overnight. After incubation, the coating solution was removed and the flasks were washed twice with milliQ $\mathrm{H}_{2} \mathrm{O}$. If not used immediately, the coated flasks were dried and stored at $4^{\circ} \mathrm{C}$. Astro. $4 \mathrm{U}$ and Astro.KN were cultured on Matrigel (Corning, USA) coating. An ice-cold suspension of 1\% Matrigel in cold DMEM/F-12 was added to cell culture plates and then incubated for at least $30 \mathrm{~min}$ at $37^{\circ} \mathrm{C}$. Before plating, Matrigel suspension was aspired.

\subsection{Organoid culture}

\section{Generation and culture of organoids}

To generate LUHMES organoids, 5000 or 7500 d2 LUHMES cells were seeded in $100 \mu \mathrm{L}$ differentiation medium per well in ULA round-bottom 96-well plates (Corning 7007, USA). To accumulate the cells at the well bottom, plates were centrifuged at 300 x g (Heraeus Multifuge 1 S-R, ThermoScientific, USA) for $5 \mathrm{~min}$. Spheres formed spontaneously. Plates were incubated at $37^{\circ} \mathrm{C}$ at $5 \% \mathrm{CO}_{2}$. Every two to three days, half the medium $(50 \mu \mathrm{L})$ was exchanged. For the generation of co-culture organoids, 4500 d2 LUHMES cells were mixed with 500 Astro.4U or 4000 d2 LUHMES cells were mixed with 1000 Astro.KN in $100 \mu \mathrm{L}$ differentiation medium per well. Generation of organoids and medium exchange was conducted in the same way as with mono-culture LUHMES organoids.

\section{Organoid plating}

To plate LUHMES or co-culture organoids, 96-well plates were coated with $50 \mu \mathrm{L} /$ well ice-cold $1 \%$ Matrigel in LUHMES differentiation medium and incubated at $37^{\circ} \mathrm{C}$ for $30 \mathrm{~min}$. Organoids were then taken up in $50 \mu \mathrm{L}$ medium with cut-off microliter pipette tips and separately seeded onto Matrigel (1 organ$\mathrm{oid} /$ well), which resulted in a Matrigel concentration of $0.5 \%$ in the well. Plates were gently rocked to ensure central placement of the organoid in the well. Plated organoids were incubated at $37^{\circ} \mathrm{C}$ with $5 \% \mathrm{CO}_{2}$.

Since Matrigel is derived from an animal source and chemically rather undefined, we tried to replace it with PLO, laminin (up to $2 \mu \mathrm{g} / \mathrm{mL}$ ), fibronectin (up to $1 \mu \mathrm{g} / \mathrm{mL}$ ) and/or collagen IV (up to $50 \mu \mathrm{g} / \mathrm{mL}$ ). However, none of these coatings led to neurite outgrowth comparable to Matrigel.

\section{Size measurement}

The size of the organoids was measured directly in the ULA round bottom plates. Bright field images of living whole organ- 
oids were recorded at 5x magnification (AxioObserver, Zeiss, Germany). The area of the organoids was evaluated by ImageJ (FIJI Version 1.51s). The threshold was determined automatically; the area was measured by the "analyze particles" algorithm with individually set low cut-off. The measured area was then calculated back to organoid diameter.

\subsection{Neurite area determination}

To determine the area of neurites that grew out of the plated organoids, plated organoids were incubated with $1 \mu \mathrm{M}$ calcein-AM (Sigma-Aldrich, USA) for $60 \mathrm{~min}$ to visualize living cells and their neurites. In an alternative approach, RFP-expressing LUHMES organoids were used and fluorescent imaging was performed (AxioObserver, Zeiss, Germany). Whole wells were imaged at $5 x$ magnification in 12 separate images that were stitched by a stitching algorithm (ZEN software blue edition, Version 1.1.2.0, Zeiss, Germany). Stitched images were processed in ImageJ (FIJI Version 1.51s). Neurites were amplified with the "find edges" algorithm. The threshold was then automatically determined by the "threshold" algorithm, and the area was measured using the "analyze particles" algorithm with appropriate cut-offs. Organoids located close to the well border were excluded from the analysis.

\subsection{Organoid viability determination}

Viability of organoids was evaluated by propidium iodide (PI, Sigma-Aldrich, USA) fluorescence intensity. Organoids were incubated with $1.5 \mu \mathrm{M}$ PI for $1 \mathrm{~h}$. Fluorescence intensity was recorded by fluorescence microscopy (AxioObserver, Zeiss, USA) and analyzed as mean grey value (MGV) in ImageJ (Version $1.51 \mathrm{~s})$. Cell death was evaluated relative to a positive control treated with $0.5 \%$ Triton-X100 (Sigma-Aldrich, USA) for $1 \mathrm{~h}$. MGVs were baseline corrected by subtracting the MGV of untreated organoids.

\subsection{Immunostaining of organoids}

To immunostain floating organoids, at least five organoids were collected in culture medium from ULA plates. Organoids were fixed in 4\% para-formaldehyde (Leica Microsystems, Germany) for $30 \mathrm{~min}$ at room temperature (RT). Fixated organoids were washed three times with PBS. After washing, organoids were permeabilized and cleared with ScaleS (20\% sorbitol, $10 \%$ glycerol, $4 \mathrm{M}$ urea, $0.2 \%$ Triton-X 100, in milliQ $\mathrm{H}_{2} \mathrm{O}$ ) (Hama et al., 2015) for $48 \mathrm{~h}$ at $4^{\circ} \mathrm{C}$. Cleared organoids were again washed three times with PBS and then blocked with blocking buffer (0.5\% Triton-X100, 5\% FBS (Gibco, USA) in PBS) at RT for $1 \mathrm{~h}$. Blocking solution was removed and primary antibodies against the proteins of interest diluted in blocking buffer were added (Tab. S1 $\left.{ }^{1}\right)$. Organoids were incubated with primary antibodies for $48 \mathrm{~h}$ at $4^{\circ} \mathrm{C}$. After incubation, organoids were washed three times with PBS. Corresponding secondary antibodies coupled to fluorophores were diluted 1:1000 in blocking buffer. Organoids were washed three times with PBS and were then incubated with secondary antibody solution for $48 \mathrm{~h}$ at $4^{\circ} \mathrm{C}$. In parallel, nuclei were counterstained with $6.15 \mu \mathrm{M}$ Hoechst-33342 (ThermoFisher, USA) in the secondary antibody solution. Phalloidin-555 (Invitrogen, USA) was applied together with primary and secondary antibodies. Organoids were then mounted on microscope slides with Aqua-Polymount (Polysciences Inc., USA) and covered with coverslips. A complete list of antibodies used is given in Tab. $\mathrm{S}^{1}$.

For immunostaining of plated organoids, organoids were plated on 8 -well $\mu$-slides (ibidi, Germany). Immunostaining was performed as described except no mounting step was performed. Plated organoids were imaged in PBS.

\subsection{Proliferation assay}

For proliferation staining, $5000 \mathrm{~d} 2$ LUHMES cells or $4500 \mathrm{~d} 2$ LUHMES with 500 Astro.4U were seeded into ULA plates for sphere generation and cultivated for up to 14 days. To visualize proliferating cells, the EdU cell proliferation kit (BaseClick, Germany) was used according to the manufacturer's instructions. The thymidine analog 5-ethynyl-2'-desoxyuridine (EdU) is only incorporated into cells in S-phase; incorporated EdU is then labeled with a fluorescent dye. Briefly, living organoids were incubated with $10 \mu \mathrm{M}$ EdU for $60 \mathrm{~min}$. Subsequently, they were fixed with 4\% para-formaldehyde for $30 \mathrm{~min}$ at RT and permeabilized with $0.5 \%$ Triton-X in PBS for $1 \mathrm{~h}$ at RT. After washing three times with PBS, 5-TAMRA-PEG3-azide (red fluorescent; $555 \mathrm{~nm}$ ) was linked to the incorporated EdU in a click reaction for $30 \mathrm{~min}$ at RT. Nuclei were either directly counterstained with $6.15 \mu \mathrm{M}$ Hoechst-33342 for $48 \mathrm{~h}$ or further immunocytochemistry was performed.

\subsection{Imaging of organoids}

To image immunostained plated and floating organoids, confocal microscopy was performed (LSM 880 with AiryScan, Zeiss, Germany). Optical sectioning was performed by acquiring z-stacks at $2 \mu \mathrm{m}$ intervals. Within one experiment, the same laser intensity was used for all samples. Light sheet images were recorded with a MuVi SPIM microscope (Luxendo, Germany). Time-lapse, bright field and epifluorescence images were recorded with an AxioObserver epifluorescence microscope (Zeiss, Germany). Images were processed in ImageJ (Fiji Version 1.51s).

\subsection{Colocalization analysis}

To evaluate the colocalization of pre- and post-synaptic markers, synaptophysin (SYP) and post-synaptic density protein 95 (PSD95) were immunostained and imaged by confocal microscopy (Zeiss LSM 880 with AiryScan,63x/1.4 Plan Apochromat Oil objective). Mander's colocalization coefficient (Manders et al., 1993) was calculated with ImageJ's "colocalization threshold" function using Costes' randomization for threshold determination (Costes et al., 2004). Confocal z-stacks (10 $\mu \mathrm{m})$ at an interval of $0.2 \mu \mathrm{m}$ were analyzed.

\subsection{Statistical analysis}

Statistical analysis was performed with GraphPad Prism 5 software (Version 5.03). Biological replicates are indicated by "N", technical replicates are indicated by " $n$ " in the figures or figure legends. Curves were fitted by a four-parameter fit with top constraint set to $100 \%$. Statistical tests and significance levels are indicated in the figure legends. P-values $<0.05$ were regarded as statistically significant. 

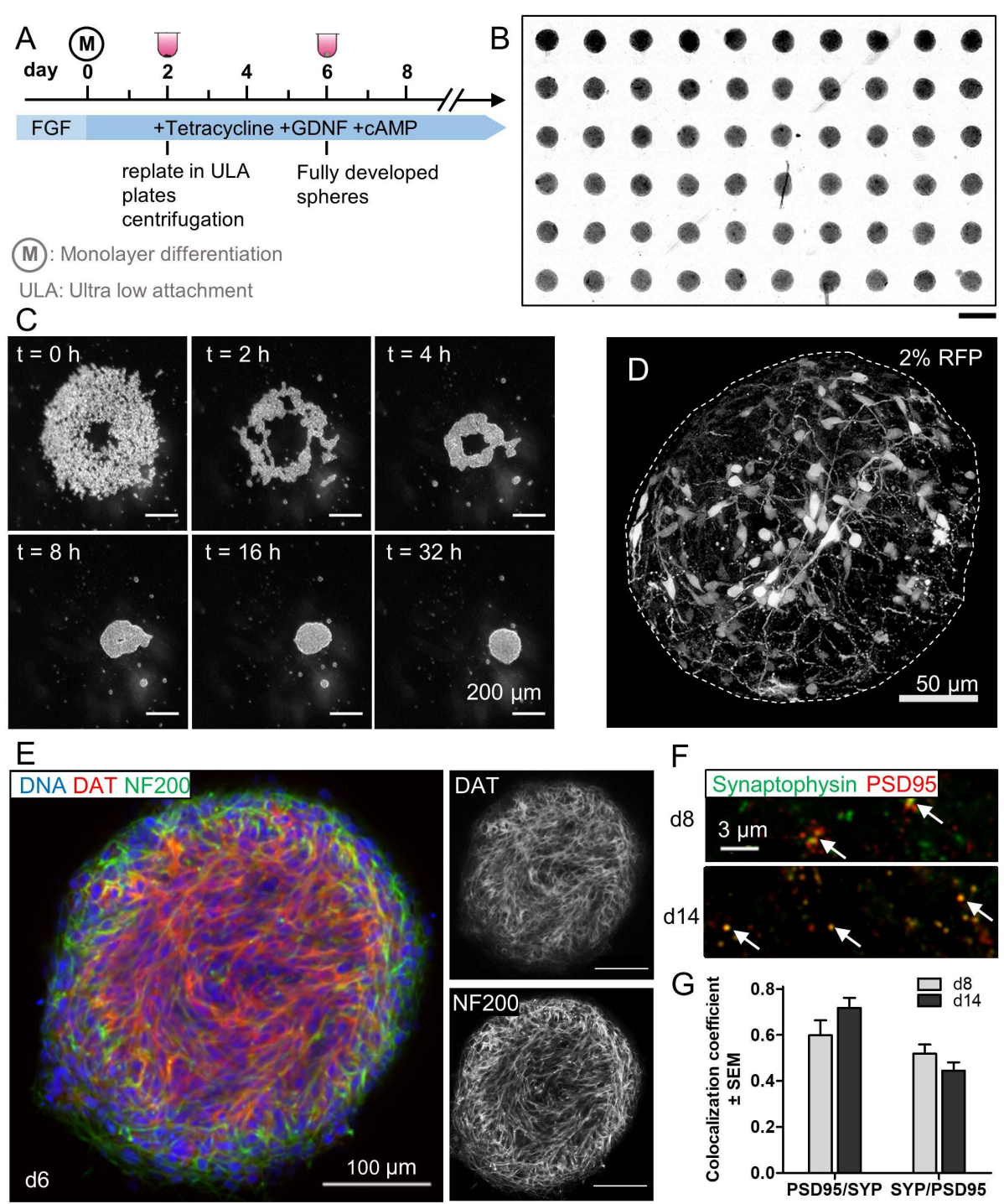

Fig. 1: Fast generation of mature neuronal organoids using a ULA plate method

(A) Generation of LUHMES organoids in ULA round bottom plates. Differentiation of LUHMES was started in monolayers (M) on d0 by supplementation of tetracycline. After $48 \mathrm{~h}$, LUHMES cells (d2, 7500 cells/well) were seeded into ULA plates and centrifuged. After $24 \mathrm{~h}$, spheres were fully formed. (B) Montage of bright field images of all 60 organoids in one ULA plate $24 \mathrm{~h}$ after seeding (d3). Scale bar $=500 \mu \mathrm{m}$. (C) Selected time points of a time-lapse recording of sphere formation in ULA plates (see video $1^{2}$ ). (D) Normal (wild-type, WT) LUHMES were mixed with LUHMES engineered to overexpress red fluorescent protein (RFP) at a WT:RFP ratio of 50:1 and used to generate neurospheres. On day 8 of differentiation, the organoids were imaged by confocal microscopy at multiple image planes in the z-dimension (z-increment: $1.31 \mu \mathrm{m}$ ). From this, a maximum intensity projection of 40 image planes was generated and displayed (total $z$ distance: $52.4 \mu \mathrm{m}$ ). The dotted line indicates the organoid border. (E) LUHMES organoids were fixed on d6 and immunostained against NF200, a pan-neuronal marker, and dopamine transporter (DAT), a marker of dopaminergic neurons. Nuclei were counterstained with Hoechst-33342. Confocal microscopy was used to visualize an image plane in the middle of the organoid. (F) Organoids were fixed and immunostained for pre-synaptic marker SYP and post-synaptic marker PSD95 on d8 and d14. Confocal detail images from inside the organoids are shown. Arrows point towards overlapping fluorescence signals, which appear yellow, indicating colocalization. (G) Colocalization of SYP and PSD95 was quantified by determining Mander's colocalization coefficient (Manders et al., 1993). It describes the relative amount of PSD95 ${ }^{+}$pixels colocalized with SYP ${ }^{+}$pixels and vice versa. Data are means \pm SEM from three organoids per time point and experiment. Experiments were conducted in independent triplicates. 


\section{Results and discussion}

\subsection{Generation and characterization of LUHMES organoids}

LUHMES organoids are structurally more similar to human brain tissue than conventional 2D cell cultures (Smirnova et al., 2016). Here, we developed a new method to generate LUHMES organoids that allows the incorporation of defined numbers of other cells, showing high reproducibility, and requires few resources and little time. First, LUHMES cells were pre-differentiated for two days, then they were seeded in defined numbers into ULA plates (Fig. 1A). After a centrifugation step, spheroids formed spontaneously within $24 \mathrm{~h}$. We recorded this process by time-lapse microscopy to visualize the kinetics of the spheroid formation (Fig. $1 \mathrm{C}$; video $1^{2}$ ). As all cells added to the well assembled to one single organoid, the method allowed the generation of highly homogeneous LUHMES organoids with control over cell numbers and size (Fig. 1B).

LUHMES 2D cultures are regarded as fully differentiated on d6 after tetracycline supplementation (Scholz et al., 2011). We therefore examined the organoids on $\mathrm{d} 6$. Immunostaining revealed a homogeneous expression of the pan-neuronal marker neurofilament 200 (NF200) and the presence of the dopamine transporter (DAT), a marker of dopaminergic neurons (Fig. 1E).

The method developed here allowed for various cell subpopulations (e.g., red fluorescent protein expressing LUHMES (RFP-LUHMES)) to be incorporated into the organoids. Spiking of the organoids with a small percentage of labelled cells allowed the visualization of single neurons inside the organoids. Confocal imaging of organoids containing $2 \%$ RFP-LUHMES on $\mathrm{d} 8$ revealed a distinct neuronal morphology of the fluorescent cells (Fig. 1D). Neither deformations of organoids nor necrotic cores were observed (Fig S3 ${ }^{1}$ ).

We further investigated the presence of other markers of neuronal maturity: Immunostaining of the pre-synaptic marker SYP and the post-synaptic marker PSD95 revealed expression of both markers. Their co-localization indicated close proximity of preand post-synaptic structures as typically formed in neuronal synapses (Fig. 1F). Quantification of this colocalization revealed an overlap of PSD95 with SYP of over $75 \%$ on d14 (Fig. 1G).

Overall, these results show that mature neuronal organoids are generated from LUHMES cells within 8 days, which is considerably faster than when generating neural organoids with stem-cell based methods. We conclude that the ULA plate method allows the easy generation of spheroids and provides the option to incorporate various cell types at defined ratios.

\subsection{Neurite outgrowth of LUHMES organoids as a functional endpoint for neurotoxicity}

Many neural organoids, including those based on LUHMES cells (Wang et al., 2018; Harris et al., 2018; Livni et al., 2019), show neurite outgrowth when plated onto an extracellular matrix. We tested whether ULA-generated LUHMES organoids behave similarly. Indeed, neurites grew out from LUHMES organoids after plating on Matrigel, while cell bodies remained localized in the organoids (Fig. 2A). Spheroids cultivated for 6-14 days prior to plating all showed neurite outgrowth, and the length of neurites (i.e., the area covered by neurites) increased continuously (Fig. 2B). Organoids plated on $\mathrm{d} 14$ reached a neurite area of $21 \pm 2 \mathrm{~mm}^{2}$ after 3 days. This corresponds to an extrapolated average neurite length of ca. $2.5 \mathrm{~mm}$. Some neurites reached a length of up to $3 \mathrm{~mm}$ before they hit the wall of the cell culture well. From this data, we calculated an average outgrowth speed of about $40 \mu \mathrm{m} / \mathrm{h}$.

To further characterize the neurites, we performed immunostaining after $72 \mathrm{~h}$. In general, neurites protruded from the organoids in a radially organized manner (Fig. 2D). The presence of growth cones at the end of all neurites indicated active, ongoing neuritogenesis (Fig. 2C). Thus, this system allows the fast generation of organized neurite networks with known polarization (cell bodies in the organoids, neurites in the periphery) and a clear spatial separation of cell bodies and neurites. Here, we refer to plated organoids as 2.5 dimensional (2.5D) cultures.

To enable testing of toxicants, we established a viability endpoint for LUHMES organoids based on the uptake of propidium iodide (PI). The dye enters only dead cells with damaged cell membranes and stains their DNA (Riccardi and Nicoletti, 2006). In a first experiment, we evaluated the fluorescence intensity of whole, floating organoids stained with PI as a measure for cell death. With increasing concentrations of $\mathrm{MPP}^{+}$, the fluorescence of PI increased, whereas the intensity of the live cell stain calcein decreased (Fig. 3A). Changes occurred exactly in the concentration range relevant for MPP ${ }^{+}$toxicity (Schildknecht et al., 2009). Therefore, this endpoint allowed the assessment of overall cell viability in organoid structures, which do not easily allow single cell analysis.

From previous studies, we know that some neurotoxicants may exert specific effects on neurites without killing the cells (Stiegler et al., 2011; Krug et al., 2013; Delp et al., 2018b). However, the assessment of neurite integrity in $3 \mathrm{D}$ cultures is a major challenge: It requires intricate staining and imaging protocols, and it requires $3 \mathrm{D}$ rendering or stereology procedures. This makes it hard to assess large sample numbers, e.g., for substance screening. The outgrown neurites of $2.5 \mathrm{D}$ cultures are more accessible to staining and imaging than neurites inside organoids, while their somata remain in a $3 \mathrm{D}$ environment. Thus, we aimed to utilize the neurite outgrowth of LUHMES organoids as an endpoint for neurotoxicological testing. To this end, we established an exposure scheme based on the treatment of LUHMES organoids. Exposure to the dopaminergic neuron-specific toxicant $\mathrm{MPP}^{+}$for $72 \mathrm{~h}$ during neurite outgrowth allowed us to quantify the neurite area of the same organoids every $24 \mathrm{~h}$ (Fig. S4 ${ }^{1}$ ). The test compound drastically reduced the neurite area after $72 \mathrm{~h}$ exposure. Furthermore, the MPP + effect was completely rescued by GBR-12909, a DAT inhibitor. These data add further evidence of the dopaminergic phenotype of LUHMES cells, and they suggest the suitability of the organoids for toxicity testing.

From this pilot experiment, we developed an exposure scheme for toxicity testing: LUHMES organoids were plated on $\mathrm{d} 8$ and

2 doi:10.14573/altex.1911111s2 


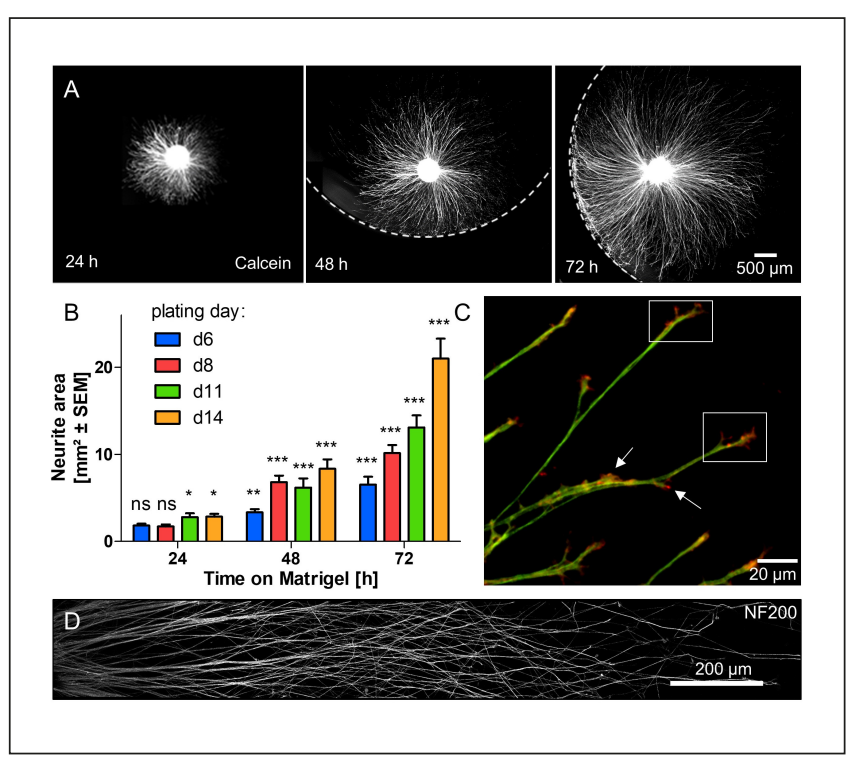

Fig. 2: Rapid and extensive neurite outgrowth from organoids after plating

(A) LUHMES organoids were transferred on d8 from ULA plates to 96 -well dishes coated with $0.5 \%$ Matrigel. After 24, 48 and $72 \mathrm{~h}$ they were live-stained with calcein-AM and imaged by fluorescence microscopy. Images representative of five organoids per time point are displayed. The dashed line indicates the edge of the cell culture dish. (B) The total area of neurite outgrowth was determined for different plating days for five organoids per time point. Plated organoids were live-stained (calcein-AM) and imaged by fluorescence microscopy. Images were analysed in ImageJ. The experiment was performed in three independent biological replicates. Data are means \pm SEM. Statistical significance was evaluated by two-way ANOVA with Bonferroni's post hoc test. Asterisks above bars indicate significance vs the sphere-covered area $24 \mathrm{~h}$ after plating. ${ }^{*} p<0.05,{ }^{* *} p<0.01,{ }^{* * *} p<0.001$. (C) LUHMES organoids were plated on d14 and fixed after $72 \mathrm{~h}$. F-actin was stained with phalloidin-555 and $\beta 3$-tubulin with a TUJ1 antibody. A fluorescence microscopy image of the growth cones of neurites is shown. White boxes indicate areas with growth cones; white arrows indicate emerging branches. Growth cones are characterized by a $\beta 3$-tubulin-negative and strongly F-actin positive broadened tip. (D) LUHMES organoids were plated on d8 and fixed on d11. A representative, confocal image of immunostained (NF200) neurites is shown. The organoid is located to the left (not visible).

subsequently treated with the substance of interest for $72 \mathrm{~h}$ (Fig. 3B). Calcein/PI double staining was used to assess toxicant effects on the $2.5 \mathrm{D}$ cultures. This method allowed the parallel evaluation of neurite outgrowth (calcein-positive) and cell viability (PI-positive) by automated high-content imaging (Fig. 3C). Treatment of plated organoids during neurite outgrowth with as little as $1 \mu \mathrm{M} \mathrm{MPP}+$ revealed a decreased neurite area while general cell viability was not affected. This indicates a specific toxicity of $\mathrm{MPP}^{+}$for neurites extending from organoids (Fig. 3D). Moreover, the potency of $\mathrm{MPP}^{+}$(low $\mu \mathrm{M}$ range) underlines the exquisite sensitivity of this test system for toxicants relying on the dopaminergic phenotype (DAT expression) of target cells. Typical concentrations of $\mathrm{MPP}^{+}$used in other test systems range in the mM range (Kim and Park, 2018; Fonck and Baudry, 2001).

To evaluate the specificity of our assay, we tested four other compounds that earlier were identified to affect the neurite outgrowth of 2D LUHMES (Delp et al., 2018b). Moreover, the proteasome inhibitor MG132 was included as a neurodegenerative compound that has no selectivity for neurites (Gutbier et al., 2018b). In good agreement with published data on 2D LUHMES, MPP ${ }^{+}$, rotenone, carbaryl, valinomycin and colchicine all caused reduced neurite outgrowth at concentrations that did not kill the cells. As expected, MG132 affected neurites only at concentrations that killed the cells (Fig. 3E). These results show that we can detect specific neurite toxicants with LUHMES organoids by an automated imaging approach. The high content imaging method allows high throughput compound testing. In summary, the $2.5 \mathrm{D}$ organoid approach allows easy assessment of neurite-specific toxicity, while it provides opportunities for extended exposures and close interactions of cell bodies typical for organoid systems.

Moreover, this spatial separation of somata and neurites allows the investigation of neurite-specific processes that are not trivial in complex networks of somata/neurites found in conventional cultures: Here, we exemplified this advantage by tracking mitochondrial movement in the outgrown neurites of plated LUHMES organoids. Somatic mitochondria interfere with image analysis of neurite-located mitochondria, limiting the valid fields for image analysis. The 2.5D configuration allowed tracking mitochondria exclusively located in neurites without actively needing to avoid imaging of somata. This analysis allowed quantification of mitochondrial speeds and confirmation of earlier findings that mitochondrial toxicants $\left(\mathrm{MPP}^{+}\right.$, rotenone) reduce mitochondrial mobility prior to effects on cell viability or neurite structures (Fig. S5 ${ }^{1}$ ).

\subsection{Incorporation of human stem-cell derived astrocytes into LUHMES organoids}

The human brain consists of many cell types besides neurons. In particular, astrocytes have gained increasing attention (Chung et al., 2015; Li et al., 2019; Nedergaard et al., 2003; Falsig et al., 2008; Ji et al., 2019; Clarke and Barres, 2013; Verkhratsky and Nedergaard, 2018). Co-culture systems are very attractive to study effects of astrocytes on neurons. We therefore investigated whether astrocytes can be incorporated into LUHMES organoids. To this end, we mixed LUHMES with iPSC-derived human astrocytes (Astro.4U) and seeded the cell suspension into ULA plates.

Spheroids were formed that were similar to LUHMES mono-culture organoids (Fig. 4A), and astrocytes were well integrated (Fig. 4B; videos $2^{3}$ and $3^{4}$ ). Intriguingly, the continued

\footnotetext{
3 doi:10.14573/altex.1911111s3

4 doi:10.14573/altex.1911111s4
} 

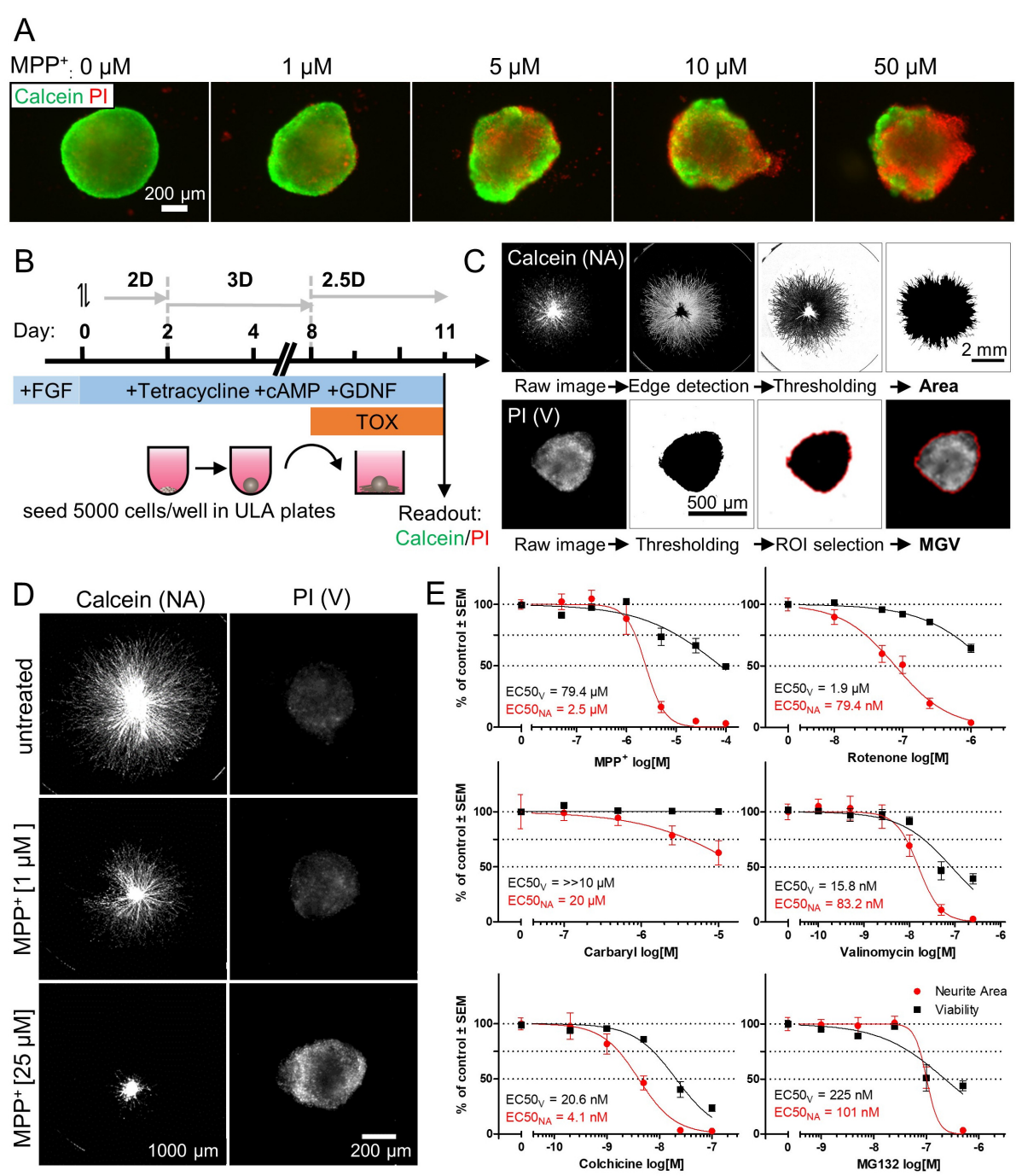

Fig. 3: Identification of specific neurotoxicants by utilizing the neurite outgrowth of LUHMES organoids

(A) LUHMES organoids (non-attached) were treated with MPP ${ }^{+}$in ULA plates for $72 \mathrm{~h}$. Subsequently, they were live-stained with calcein$\mathrm{AM}$ and PI. Representative fluorescent microscopy images are shown. (B) LUHMES organoids were plated on Matrigel on d8. After $2 \mathrm{~h}$ of attachment, they were treated for $72 \mathrm{~h}$. On d11, they were live-stained with PI and calcein-AM. (C) ImageJ workflow to determine neurite area (NA) and viability (V): The total NA was determined from whole-well images of calcein-stained neurites. $V$ was determined by the overall PI fluorescence intensity (mean grey value, MGV) relative to a dead control (0.5\% Triton-X100 for $1 \mathrm{~h}$ ). (D) Representative images of neurite outgrowth (stained with calcein) and PI fluorescence of plated organoids treated with MPP ${ }^{+}$for $72 \mathrm{~h}$ (d8-d11). Images were recorded by fluorescent microscopy. (E) Concentration-response curves of example substances tested in the assay described in $\mathrm{D}$. The neurite area was normalized to a solvent control; viability was normalized to a dead control (0.5\% Triton-X100). EC50 NA and EC50v were determined from the curve fits. Data are means \pm SEM of three biological replicates. For each biological replicate, at least three organoids were analyzed per condition.

growth in mono-culture spheres was not observed for LUHMESastrocyte mixed organoids (Fig. 4C). To test whether astrocytes prevented proliferation of LUHMES cells in organoids, we performed an EdU proliferation assay, which revealed proliferating cells in LUHMES organoids as described earlier (Smirnova et al., 2016), but not in co-culture organoids (Fig. 4D). This shows that incorporation of astrocytes into LUHMES organoids leads to the formation of a stable, post-mitotic system. Mono-culture LUHMES proliferation alternatively could be prevented by $48 \mathrm{~h}$ treatment with taxol (Smirnova et al., 2016) or cytarabine (AraC) (Fig. S6 ${ }^{1}$ ). However, incorporation of astrocytes seems to be more physiological than treatment with cytostatic drugs, and it prevents possible interferences of the substance with neurite outgrowth or other endpoints. 

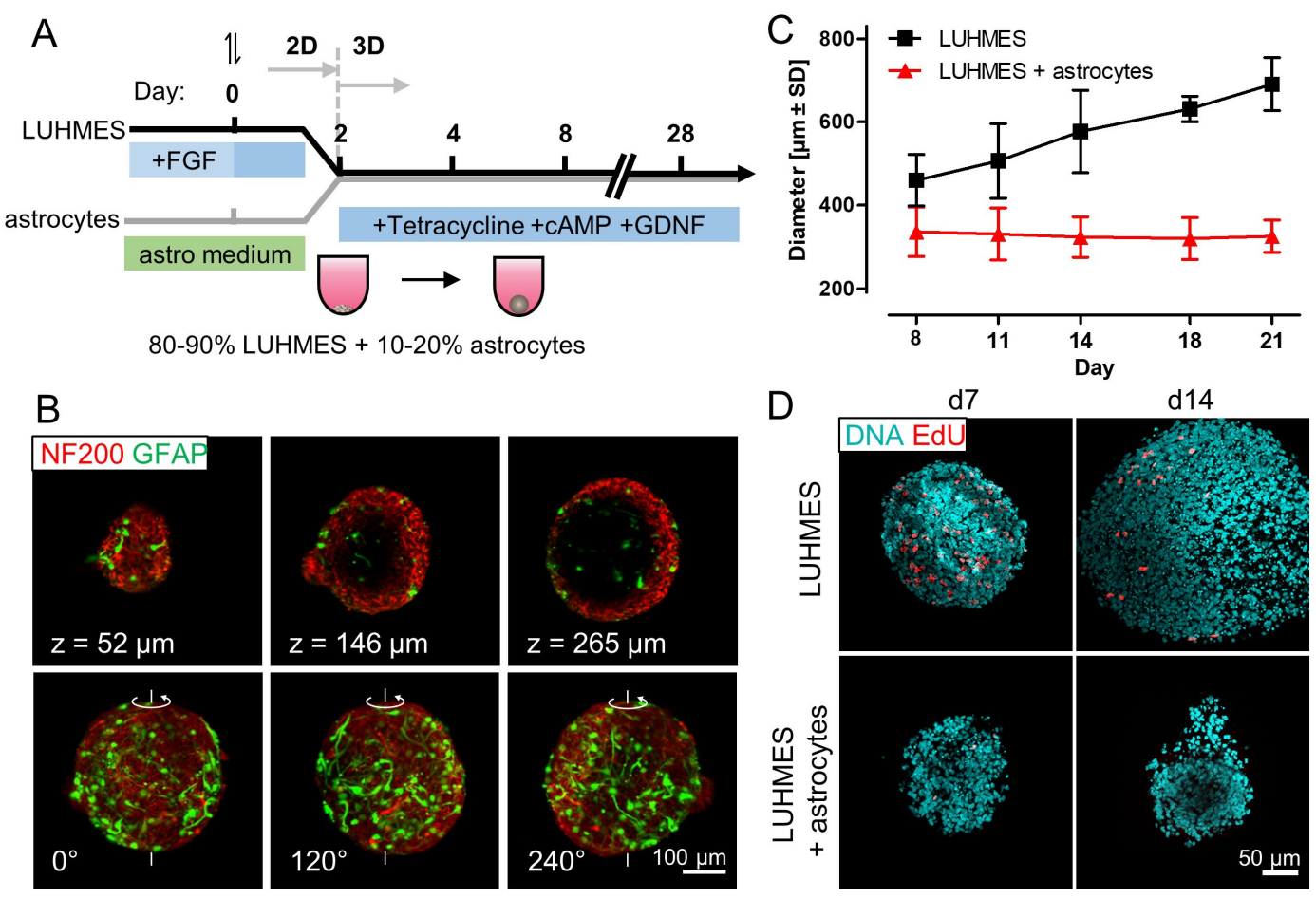

Fig. 4: Stabilization of LUHMES organoids by incorporation of stem cell-derived human astrocytes

(A) Co-culture spheres were generated by mixing d2 LUHMES and astrocytes (Astro.4U 10:1, Astro.KN 5:1) and seeding 5000 cells/well into ULA-plates. After centrifugation, spheres formed spontaneously within $24 \mathrm{~h}$. (B) Co-culture organoids (10\% Astro.4U) were fixed and immunostained on $\mathrm{d} 6$. Light sheet microscopy was performed, and 3D reconstruction images were rendered. The upper row shows three different $z$-planes from the pole to the equator of the sphere. The lower row shows different perspectives. A full rotation of the sphere can be seen in video $2^{3}$. $0^{\circ} \triangleq 0 \mathrm{~s}, 120^{\circ} \triangleq 1.6 \mathrm{~s}, 240^{\circ} \triangleq 3.2 \mathrm{~s}$. (C) Size of LUHMES and co-culture organoids (10\% Astro.4U) was measured during three weeks of cultivation by bright field imaging and subsequent image analysis. Data are means $\pm S D(n=10, N=3)$. (D) 5-Ethynyl-2'-deoxyuridine (EdU) was added for $60 \mathrm{~min}$ on d7 or d14. Staining for incorporated EdU was used to visualize proliferating cells in mono- and co-culture organoids. Nuclei were counterstained with Hoechst- $33342.12 \pm 5 \%$ of cells in LUHMES organoids were found to be EdU positive on d8. In coculture organoids, only single cells $(<1 \%)$ were EdU-positive. Organoids were imaged by confocal microscopy with a $2 \mu \mathrm{m} z$-increment. Maximum z-projections of $10 \mu \mathrm{m}$ of the organoids are shown.

After three weeks in culture, most organoids contained areas in which astrocyte processes and neurites were enriched relative to cell bodies (Fig. 5A). Future studies may attempt to incorporate oligodendrocytes and to examine whether such areas form correlates of "white matter".

Immunostaining of astrocyte-LUHMES organoids, cultured for up to 4 weeks revealed structural integrity throughout, and absence of any nuclear fragmentation. After four weeks, astrocytes were still fully integrated in the organoids. When these co-culture organoids were plated, LUHMES cells showed the radial outgrowth observed in mono-culture organoids. Moreover, astrocytes organized radially around the organoid, forming a glial corona (Fig. 5B).

We tested whether astrocytes from different sources behave similarly (Chandrasekaran et al., 2016) by using an established gliogenesis protocol to produce astrocytes from pluripotent stem cells (Palm et al., 2015). These glia cells (termed Astro.KN) integrated well, prevented LUHMES organoid growth (Fig. S71), and enabled long term culture (Fig. 5C). They also formed a corona around the organoid upon plating (Fig. 5D).

These experiments show that our modular approach for organoid generation can be utilized to integrate different types of astrocytes into LUHMES organoids at defined ratios (e.g., 10\% Astro. $4 \mathrm{U}$ or $20 \%$ Astro.KN). Co-culture with astrocytes leads to a general stabilization of the system, allowing long-term culture.

To make the system more broadly available, we tested whether organoids can be shipped. For this, we used overnight mail and stored organoids in sealed ULA plates. After arrival, organoids showed normal viability and neurite outgrowth after plating (Fig. S8A ${ }^{1}$ ). Shipped plated organoids were used to investi- 

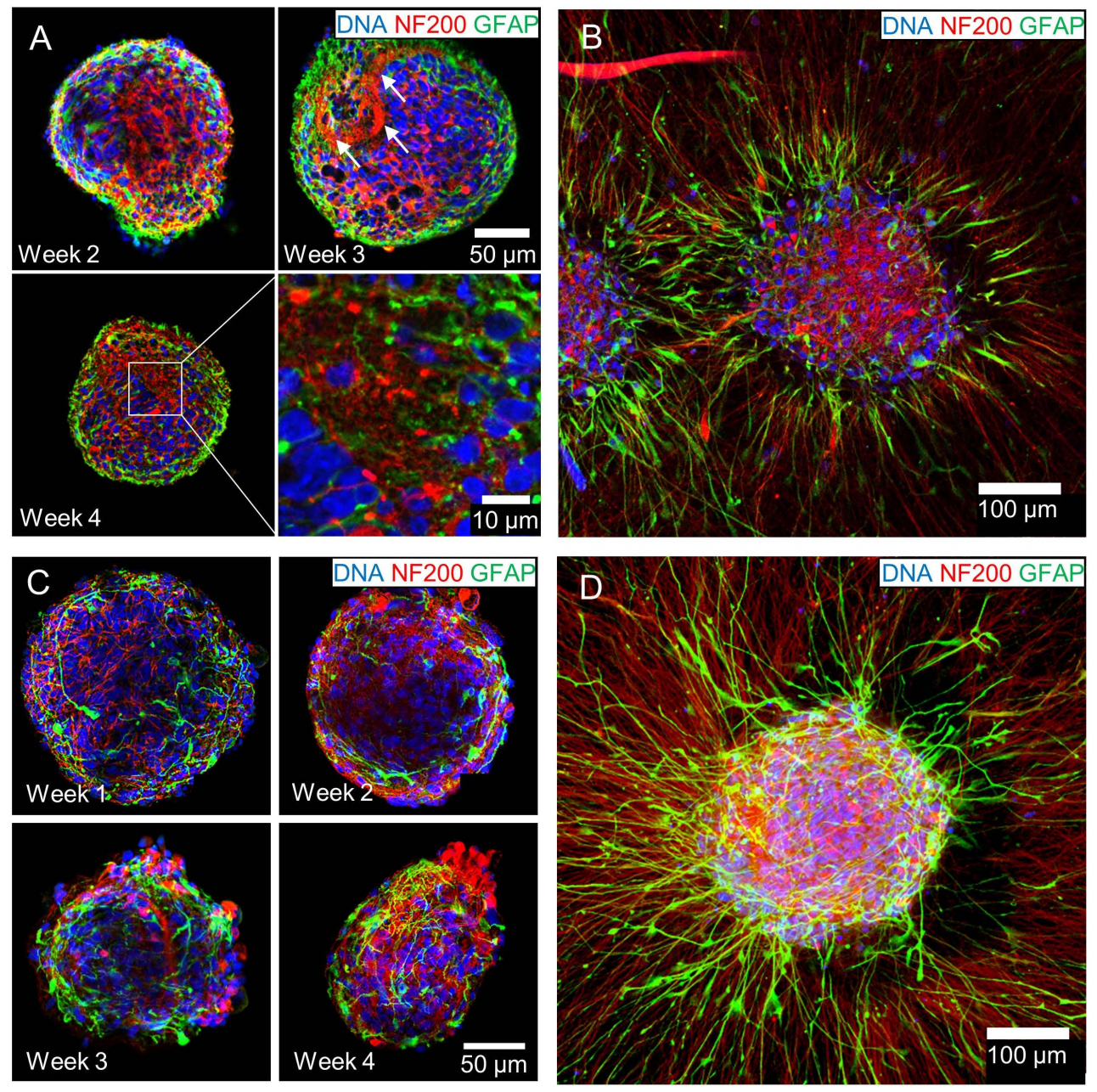

Fig. 5: Neuro-glial interaction upon long-term culture and plating of mixed organoids

(A) Co-culture organoids with $10 \%$ Astro.4U were fixed on d14, d21 and d28 and immunostained against GFAP and NF200. Nuclei were counterstained with Hoechst-33342. Optical sections in the middle of the organoids are shown. A dense, nuclei-free area is indicated with arrows, and is shown in detail below. (B) On d8, co-culture organoids were plated on a Matrigel coated plate. They were fixed and immunostained after $72 \mathrm{~h}$. A $10 \mu \mathrm{m}$ z-projection of confocal optical sections is shown (z-increment: $2 \mu \mathrm{m})$. (C) Confocal optical sections of co-culture organoids with $20 \%$ Astro. KN in the organoids. Organoids were fixed and immunostained after 1, 2, 3 or 4 weeks in culture.

(D) Z-projection of plated co-culture organoids with $20 \%$ Astro.KN after $72 \mathrm{~h}$ on Matrigel. z-increment: $2 \mu$ m; maximum z-projection: $26 \mu$ m.

gate the functional electrophysiological properties of this system. Increases in intracellular free calcium concentrations could be measured with a calcium-sensitive fluorescent dye after depolarization with $\mathrm{KCl}, \mathrm{Na}^{+}$-channel opening with veratridine, purinergic receptor triggering with $\alpha, \beta$-meATP, and ionotropic acetylcholine receptor triggering with nicotine (Fig. S8B ${ }^{1}$ ). Co-culture organoids also showed spontaneous activity when plated on micro-electrode array (MEA)-chips. However, only electrodes directly covered by the soma aggregation of the organoid showed spontaneous spikes and bursts, and future optimization or change of the MEA platform will be required (Fig. $8 \mathrm{C}^{-E^{1}}$ ). The data suggest further uses of LUHMES organoids for studies of electrophysiological disturbances by toxicants.

\subsection{Neuroprotection by astrocytes in co-culture organoids}

One major function of astrocytes is neuroprotection. Uncovering modes of astrocyte-mediated neuroprotection might provide the basis for new strategies of neuroprotection (Hansson et al., 2000). Moreover, the neuroprotective effect of astrocytes could modu- 
A

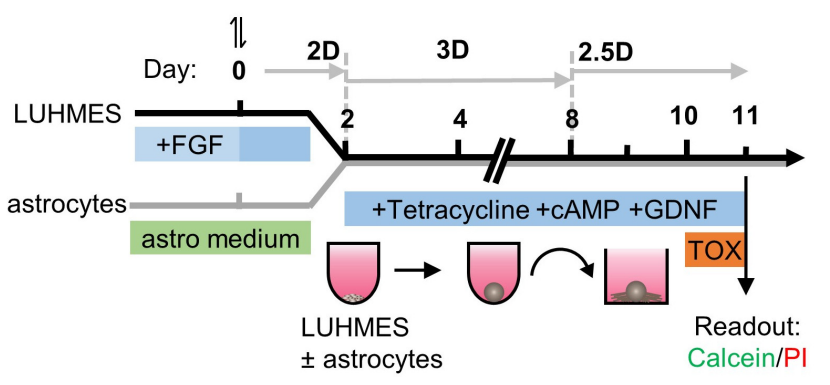

C

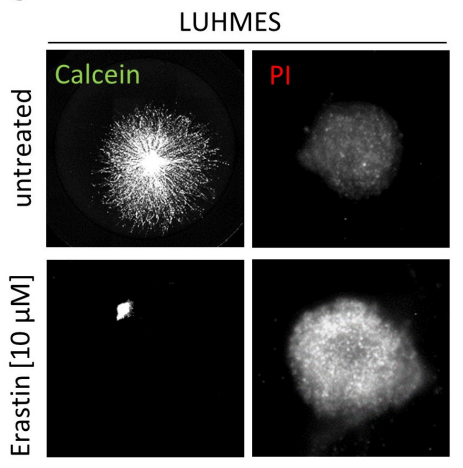

LUHMES + astrocytes

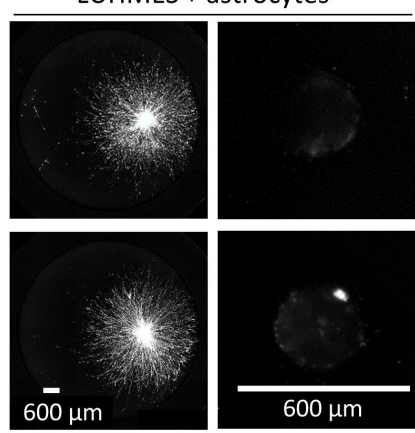

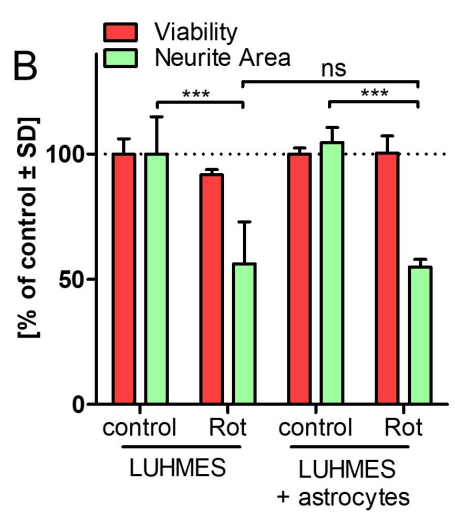

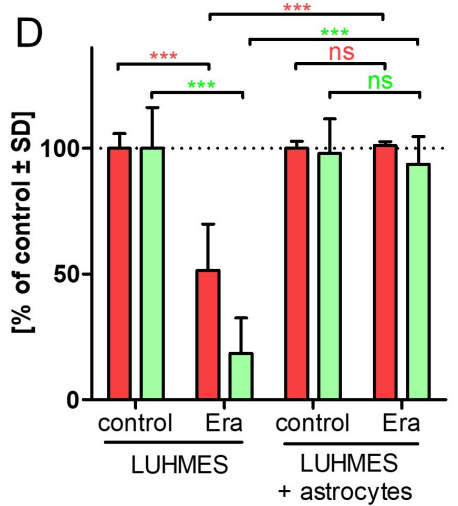

Fig. 6: Neuroprotection by co-cultured astrocytes from erastin-induced ferroptosis

(A) Co-culture and LUHMES organoids were generated in ULA plates (5000 cells/well). LUHMES and co-culture organoids with $20 \%$ Astro. KN were transferred to Matrigel-coated 96 -well plates on d8. After $48 \mathrm{~h}$ of neurite outgrowth, they were treated with erastin or rotenone for $24 \mathrm{~h}$. Plated organoids were then live-stained with calcein-AM and PI. (B) Whole well images of calcein-stained organoids were used to quantify the total neurite covered area of LUHMES mono-culture and co-culture organoids at $72 \mathrm{~h}$ after plating with or without the $24 \mathrm{~h}$ treatment with rotenone (Rot, $1 \mu \mathrm{M})$. PI staining was used to quantify the viability of organoids. The mean PI- fluorescence was normalized to a dead control (organoids treated with $0.5 \%$ Triton-X100). (C) Representative fluorescence images of calcein- and Pl-stained organoids after $24 \mathrm{~h}$ of erastin treatment. (D) Neurite area and viability of organoids was quantified after $24 \mathrm{~h}$ treatment with erastin (Era, $10 \mu M)$. $(n=3, N=3)$. Statistical significance was determined by one-way ANOVA with Tukey's post hoc multiple comparisons test. $n s=$ not significant. ${ }^{* *} p<0.001 . n \geq 3$. Data are means $\pm S D$.

late the adverse effects of potential neurotoxic compounds (Pizzurro et al., 2014; Gutbier et al., 2018b; Gerhardt et al., 2001). However, the role of glia still needs to be defined for several recently discovered modes of neuronal death.

For instance, little is known about ferroptosis, an iron-dependent mode of programmed cell death (Dixon et al., 2012; Guiney et al., 2017). To investigate the potential protective role of astrocytes in this type of neurodegeneration, we plated LUHMES mono- and co-culture organoids and treated them for $24 \mathrm{~h}$ with the canonical ferroptosis inducer erastin (Wolpaw et al., 2011; Dolma et al., 2003; Dixon et al., 2012) (Fig. 6A). Neurite outgrowth was used as test endpoint. In mono-cultures in $2.5 \mathrm{D}$ format, drastic reduction in neurite area and pronounced cytotoxicity were observed. However, there was no significant decrease in neurite area or cell viability in the presence of astrocytes (Fig. 6C,D). The glial cells protected the neurons against erastin concentrations as high as $40 \mu \mathrm{M}\left(4 \mathrm{x}\right.$ higher than the $\left.\mathrm{IC}_{50}\right)$ (Fig. S9 $\left.{ }^{1}\right)$.

For comparison, non-ferroptotic neuronal stress was triggered by rotenone (Smirnova et al., 2016; Harris et al., 2018). Under such conditions, a specific reduction of the neurite area was observed, but cell bodies survived. In this paradigm, we observed no protection by the astrocytes (Fig. 6B).

As a third neuronal death model, we inhibited the proteasome by incubation with MG132 for $24 \mathrm{~h}$. In earlier studies, we had observed that astrocytes protect neurons against proteasome inhibition by supplying thiol (Gutbier et al., 2018b). Using LUHMES organoids in the $2.5 \mathrm{D}$ format (Fig. 7A), we observed severe neurodegeneration triggered by MG132 in mono-culture organoids, 
A

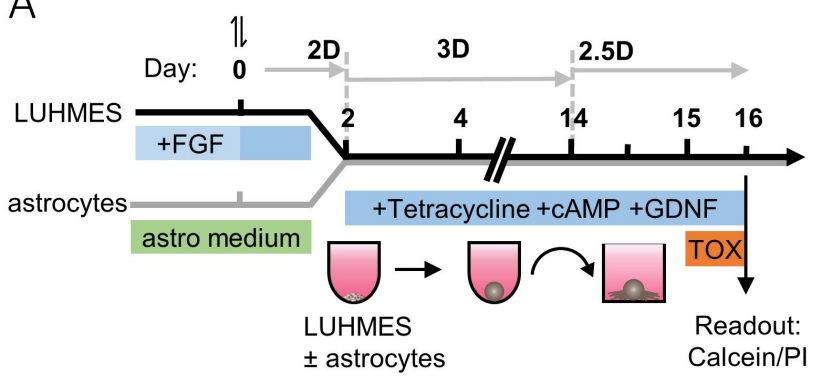

B
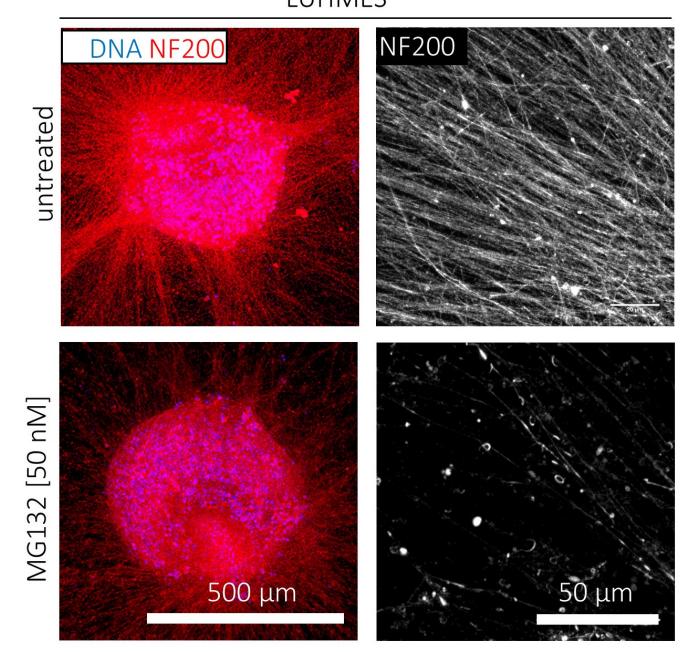
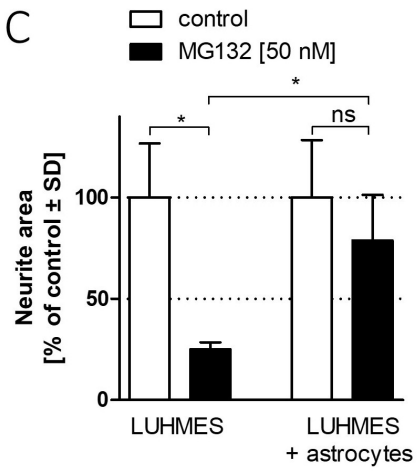

LUHMES + astrocytes
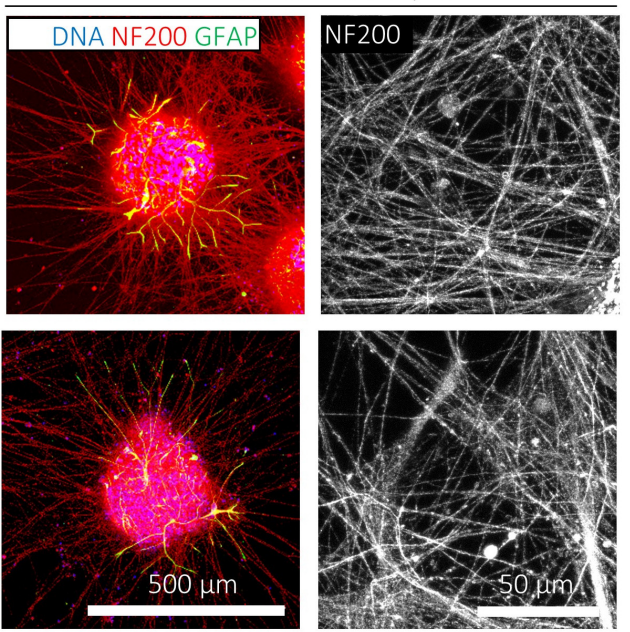

Fig. 7: Protection of neurons by astrocytes from degeneration mediated by proteasome inhibition

(A) LUHMES and co-culture spheres (10\% Astro.4U) were plated on Matrigel on d14 for $72 \mathrm{~h}$. Treatment with the proteasome inhibitor MG132 was conducted for $24 \mathrm{~h}$ from d16 to d17; then spheres were fixed and immunostained. (B) The neurite area in direct proximity of plated organoids was quantified by evaluating NF200-positive pixels in detail images of the neurite network. Data are means \pm SD ( $n=3$ ). Statistical significance was determined by one-way ANOVA with Tukey's post hoc test. ns $=$ not significant, ${ }^{*}=p<0.05$. (C) Left: Confocal images of whole plated organoids fixed on d17 and stained for NF200 and GFAP. Right: maximum z-projections (12 $\mu \mathrm{m})$ of the neurite network (NF200) in direct proximity of plated LUHMES and co-culture organoids. z-increment: $1.21 \mu \mathrm{m}$.

but not in co-culture organoids (Fig. 7B). Quantification of the neurite area in direct proximity of the organoids (visualized by immunostaining against NF200) confirmed this observation (Fig. 7C).

This series of experiments demonstrates that the organoid composition (i.e., the presence or absence of astrocytes) may affect the outcome of neurotoxicity tests. Both erastin and MG132 have been used to model aspects of neurodegenerative diseases (Bentea et al., 2017; Sun et al., 2006; Abdalkader et al., 2018; Do Van et al., 2016). Thus, the combination of such model toxicants with an organoid test system allows new approaches to better simulate aspects of human neuropathology in vitro.

\subsection{Incorporation of microglia-like cells into organoids}

In order to test whether other types of glia may be incorporated into LUHMES organoids, we incorporated iPSC-derived GFP-expressing microglia-like cells. They were obtained by differentiation of pre-macrophages towards microglia-like cells in the presence of GM-CSF and IL-34 (Haenseler et al., 2017). The microglia were produced from iPSC that stably expressed GFP. These fluorescent cells integrated into the LUHMES organoids where they showed a ramified morphology (Fig. 8A).

Having established that LUHMES-microglia spheroids could be produced by the ULA method, we tested whether two types of glia can be combined into triple-co-culture organoids. We used a 
A
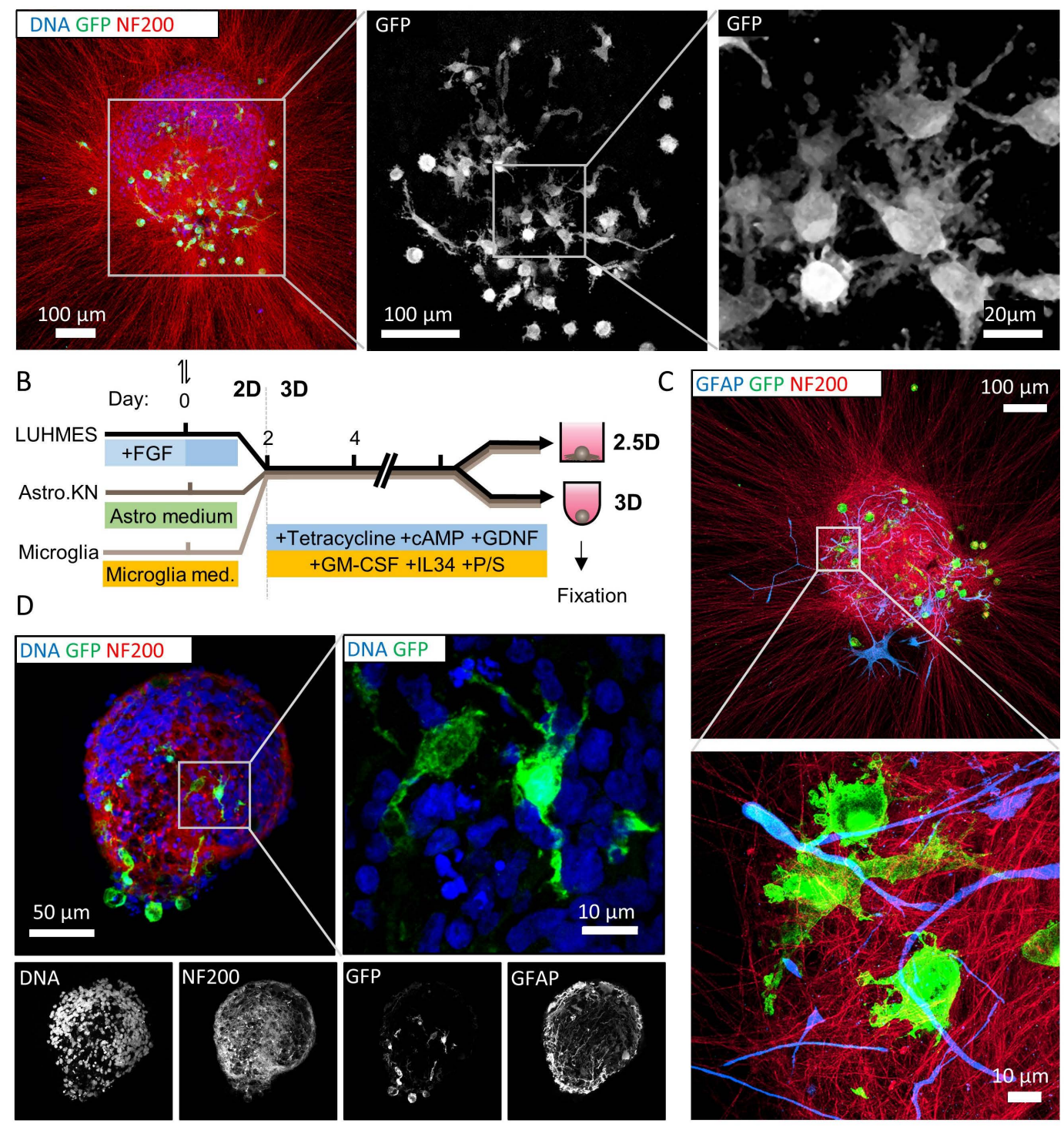

Fig. 8: Incorporation of microglia into organoids

(A) LUHMES organoids were generated from 90\% d2 LUHMES and 10\% GFP-microglia-like cells. They were plated on d11 and fixed on d14. A $50 \mu \mathrm{m}$ maximum z-projection of the immunostained plated organoids is shown (z-increment: $2 \mu \mathrm{m}$ ). Images to the right show magnifications of the regions in rectangles. (B) Triple-co-culture organoid generation scheme. On d2 of LUHMES differentiation, 5000 cells/well (80\% LUHMES, 10\% Astro.KN, 10\% GFP-microglia-like cells) were seeded into ULA round bottom plates. After centrifugation, organoids formed spontaneously within $48 \mathrm{~h}$. (C) Triple-co-culture organoids were plated on d11 and were allowed to grow out for $72 \mathrm{~h}$. Subsequently, they were fixed and immunostained for NF200, GFAP and GFP, then imaged by confocal microscopy. A Z-projection of $20 \mu \mathrm{m}$ is shown (10 optical sections separated by $2 \mu \mathrm{m}$ ). Below: Magnification of the indicated rectangle, showing ramified microglia in detail. Maximum z-projection: $20 \mu \mathrm{m}$, z-increment: $1 \mu \mathrm{m}$. (D) Floating triple-co-culture organoids were fixed on d16, immunostained and imaged by confocal microscopy. A z-projection of $24 \mu \mathrm{m}$ is shown (z-increment: $2 \mu \mathrm{m})$. Left: Composite image of the small images below. Right: Amoeboid microglia in detail. 


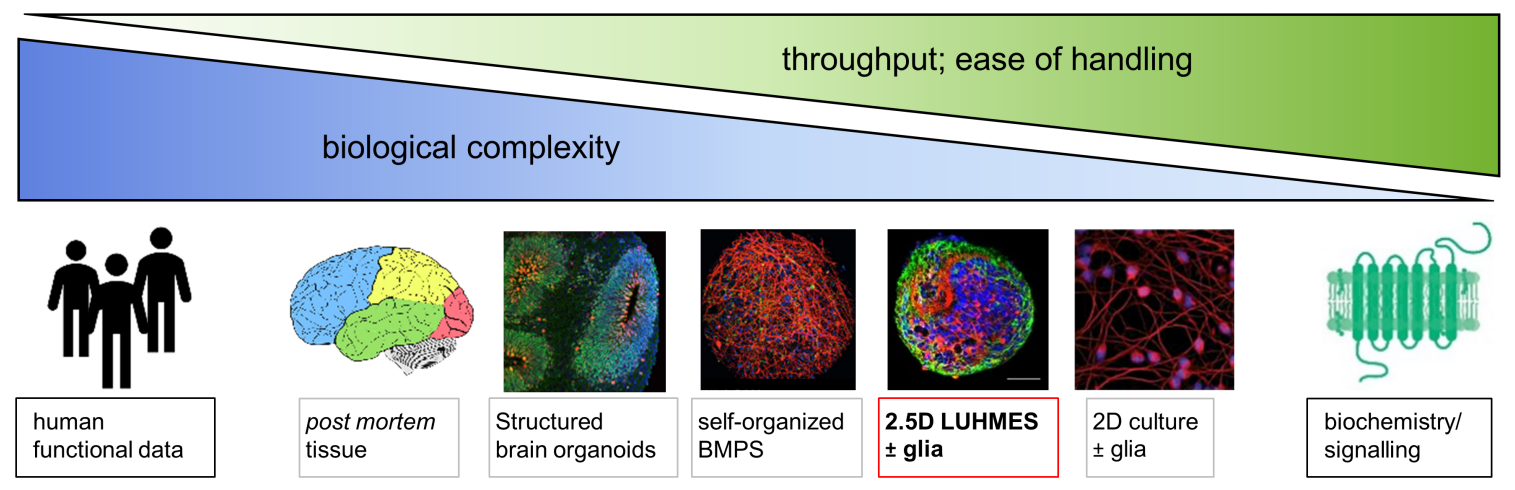

Fig. 9: Overview of neural organoid models for neurodegeneration research

Choosing the right model for a given research question is a trade-off between accessibility of the material and throughput, and complexity of the system. The latter may be linked to higher relevance with regard to in vivo predictions. Data from actual human brain serves as orientation for disease models. Tissue-mimicking brain organoids allow the close recapitulation of organizing events in brain development, and they show structural resemblance of the brain. More simple human brain microphysiological systems (BMPS) may mimic microstructures (synapses, myelin, etc.) but not overall neuronal superstructures (e.g., cortical layers). 2.5D cultures of LUHMES with human astrocytes (as presented here) combine some advantages of LUHMES cells with a more tissue-like co-cultures situation. $2 \mathrm{D}$ LUHMES culture is a well-established human neuronal model allowing high throughput and fast readouts. In screening approaches, molecular interactions between substances and bio-macromolecules such as receptors can be evaluated, but it is difficult to predict what the data means for human pathology. Images taken from: Amiri et al., 2018 (modified); Pamies et al., 2017 ; Scholz et al., 2011.

defined mix of $80 \%$ LUHMES, $10 \%$ astrocytes and 10\% microglia cells to generate spheroids in ULA plates (Fig. 8B). Here, the microglia again took on a typical ramified morphology after their incorporation in organoids (Fig. 8C). When the triple-co-culture organoids were plated, neurites grew out, but microglia remained inside the organoids. The ramified morphology of the microglia was retained after plating (Fig. 8D).

The ability to generate co-culture organoids with or without astrocytes or microglia offers a valuable tool for the investigation of neuro-glia interactions. These pilot experiments show that LUHMES organoids can be further refined by the incorporation of microglia at defined ratios.

\section{Conclusions and outlook}

Here, we introduce a new method to generate LUHMES organoids with or without glia. The ULA round-bottom plate approach allows a fast and reproducible generation of mature neuronal organoids. Concerning neurotoxicity endpoints, we demonstrated the usefulness of a rapid and simple PI/calcein staining protocol. More importantly, we introduce the use of so-called 2.5D cultures, in which reduced neurite outgrowth can be measured as an endpoint for specific neurotoxicity. By co-culturing
LUHMES with stem cell-derived human astrocytes in $3 \mathrm{D}$, post-mitotic organoids were formed without the need for adding mitosis inhibitors. The co-culture organoids proved suitable for studies of neurotoxicity and neuroprotection. It is of particular interest that they may be used as a human cell-based model to study ferroptosis. In preliminary experiments, we also demonstrated that the organoid system can be expanded by adding further cell types such as microglia.

In vitro modelling of the brain necessitates a trade-off between approaching the brain's complexity and being able to obtain quantifiable readouts (Fig. 9). For instance, post mortem tissue may be used to study the brain, but respective test systems allow only very low throughput. On the other end of the spectrum of models for human neurodegeneration, studying receptors of human neurons (Sipes et al., 2013) allows a high throughput, but it is difficult to extrapolate results to whole tissues or patients. In vitro assays using human neurons represent a compromise between molecular approaches and post mortem tissue. These systems allow high throughput when high-content imaging and automated image evaluation are used as endpoints. However, conventional cell monolayers fail to recapitulate important features of the brain, such as the tight packing of cells in a tissue structure. Organoid cultures may be able to bridge this gap. The most advanced systems are able to recapitulate brain tissue architecture, 
like cortical layering (Lancaster et al., 2013, 2017). Others show less sophisticated patterning, but contain most cell types present in the brain (Pamies et al., 2017). Building these organoids requires lengthy protocols, and toxicity readouts are difficult to implement. Additionally, the multitude of cell types - while closely modelling the in vivo situation - complicates the analysis of toxicity mechanisms. Homogeneous mono-culture organoids, such as 3D LUHMES (Smirnova et al., 2016), allow mechanistic studies, e.g., on cellular resilience (Harris et al., 2018).

An expansion of this model system is the organoid co-culture of LUHMES and stem-cell derived human astrocytes (with or without microglia). Integration of glia allows the generation of post-mitotic organoids suitable for long-term culture. The modularity of the system facilitates the study of glial effects, as demonstrated here by their protection of neurons against erastin and MG132. The additional incorporation of microglia opens up new opportunities for studies of neuroinflammation. This three-celltype modularity could also allow modelling the cellular composition of different brain regions, which may help to identify why some brain regions are more severely affected by neurodegenerative diseases than others.

Due to the LUHMES cells' dopaminergic phenotype and the organoids' responsiveness to model toxicants $\left(\mathrm{MPP}^{+}, \mathrm{MG} 132\right.$, rotenone), application of our system as an animal-free in vitro model for Parkinson's disease is of particular interest.

\section{References}

Abdalkader, M., Lampinen, R., Kanninen, K. M. et al. (2018). Targeting Nrf2 to suppress ferroptosis and mitochondrial dysfunction in neurodegeneration. Front Neurosci 12, 466. doi:10.3389/fnins.2018.00466

Amiri, A., Coppola, G., Scuderi, S. et al. (2018). Transcriptome and epigenome landscape of human cortical development modeled in organoids. Science 362, eaat6720. doi:10.1126/science. aat6720

Barbosa, D. J., Capela, J. P., de Lourdes Bastos, M. et al. (2015). In vitro models for neurotoxicology research. Toxicol Res 4, 801-842. doi:10.1039/C4TX00043A

Bélanger, M. and Magistretti, P. J. (2009). The role of astroglia in neuroprotection. Dialogues Clin Neurosci 11, 281-295.

Bentea, E., Verbruggen, L. and Massie, A. (2017). The proteasome inhibition model of Parkinson's disease. J Parkinsons Dis 7, 31-63. doi:10.3233/JPD-160921

Bylicky, M. A., Mueller, G. P. and Day, R. M. (2018). Mechanisms of endogenous neuroprotective effects of astrocytes in brain injury. Oxid Med Cell Longev 2018, 6501031-6501031. doi:10.1155/2018/6501031

Camp, J. G., Badsha, F., Florio, M. et al. (2015). Human cerebral organoids recapitulate gene expression programs of fetal neocortex development. Proc Natl Acad Sci US A 112, 1567215677. doi:10.1073/pnas.1520760112

Chandrasekaran, A., Avci, H. X., Leist, M. et al. (2016). Astrocyte differentiation of human pluripotent stem cells: New tools for neurological disorder research. Front Cell Neurosci 10, 215. doi:10.3389/fncel.2016.00215
Chen, P. C., Vargas, M. R., Pani, A. K. et al. (2009). Nrf2-mediated neuroprotection in the MPTP mouse model of Parkinson's disease: Critical role for the astrocyte. Proc Natl Acad Sci US A 106, 2933-2938. doi:10.1073/pnas.0813361106

Chung, W.-S., Allen, N. J. and Eroglu, C. (2015). Astrocytes control synapse formation, function, and elimination. Cold Spring Harb Perspect Biol 7, a020370. doi:10.1101/cshperspect. a020370

Clarke, L. E. and Barres, B. A. (2013). Emerging roles of astrocytes in neural circuit development. Nat Rev Neurosci 14, 311321. doi: $10.1038 /$ nrn3484

Costes, S. V., Daelemans, D., Cho, E. H. et al. (2004). Automatic and quantitative measurement of protein-protein colocalization in live cells. Biophys $J$ 86, 3993-4003. doi:10.1529/biophysj. 103.038422

Delp, J., Gutbier, S., Cerff, M. et al. (2018a). Stage-specific metabolic features of differentiating neurons: Implications for toxicant sensitivity. Toxicol Appl Pharmacol 354, 64-80. doi:10.1016/j.taap.2017.12.013

Delp, J., Gutbier, S., Klima, S. et al. (2018b). A high-throughput approach to identify specific neurotoxicants/ developmental toxicants in human neuronal cell function assays. ALTEX 35, 235-253. doi:10.14573/altex.1712182

Delp, J., Funke, M., Rudolf, F. et al. (2019). Development of a neurotoxicity assay that is tuned to detect mitochondrial toxicants. Arch Toxicol 93, 1585-1608. doi:10.1007/s00204-01902473-y

Dezonne, R. S., Sartore, R. C., Nascimento, J. M. et al. (2017). Derivation of functional human astrocytes from cerebral organoids. Sci Rep 7, 45091. doi:10.1038/srep45091

Dixon, S. J., Lemberg, K. M., Lamprecht, M. R. et al. (2012). Ferroptosis: An iron-dependent form of nonapoptotic cell death. Cell 149, 1060-1072. doi:10.1016/j.cell.2012.03.042

Do Van, B., Gouel, F., Jonneaux, A. et al. (2016). Ferroptosis, a newly characterized form of cell death in Parkinson's disease that is regulated by PKC. Neurobiol Dis 94, 169-178. doi:10.1016/j.nbd.2016.05.011

Dolma, S., Lessnick, S. L., Hahn, W. C. et al. (2003). Identification of genotype-selective antitumor agents using synthetic lethal chemical screening in engineered human tumor cells. Cancer Cell 3, 285-296. doi:10.1016/S1535-6108(03)00050-3

Efremova, L., Schildknecht, S., Adam, M. et al. (2015). Prevention of the degeneration of human dopaminergic neurons in an astrocyte co-culture system allowing endogenous drug metabolism. Br J Pharmacol 172, 4119-4132. doi:10.1111/ bph.13193

Efremova, L., Chovancova, P., Adam, M. et al. (2017). Switching from astrocytic neuroprotection to neurodegeneration by cytokine stimulation. Arch Toxicol 91, 231-246. doi:10.1007/ s00204-016-1702-2

Eiraku, M., Takata, N., Ishibashi, H. et al. (2011). Self-organizing optic-cup morphogenesis in three-dimensional culture. Nature 472, 51-56. doi:10.1038/nature09941

Eroglu, C. and Barres, B. A. (2010). Regulation of synaptic connectivity by glia. Nature 468, 223-231. doi:10.1038/ nature09612 
Falsig, J., Latta, M. and Leist, M. (2004). Defined inflammatory states in astrocyte cultures: Correlation with susceptibility towards CD95-driven apoptosis. J Neurochem 88, 181-193. doi:10.1111/j.1471-4159.2004.02144.x

Falsig, J., van Beek, J., Hermann, C. et al. (2008). Molecular basis for detection of invading pathogens in the brain. J Neurosci Res 86, 1434-1447. doi:10.1002/jnr.21590

Florio, M. and Huttner, W. B. (2014). Neural progenitors, neurogenesis and the evolution of the neocortex. Development 141, 2182-2194. doi:10.1242/dev.090571

Fonck, C. and Baudry, M. (2001). Toxic effects of $\mathrm{MPP}^{+}$and MPTP in PC12 cells independent of reactive oxygen species formation. Brain Res 905, 199-206. doi:10.1016/S00068993(01)02551-3

Gerhardt, E., Kugler, S., Leist, M. et al. (2001). Cascade of caspase activation in potassium-deprived cerebellar granule neurons: Targets for treatment with peptide and protein inhibitors of apoptosis. Mol Cell Neurosci 17, 717-731. doi:10.1006/ mcne.2001.0962

Gieseck III, R. L., Hannan, N. R., Bort, R. et al. (2014). Maturation of induced pluripotent stem cell derived hepatocytes by 3D-culture. PLoS One 9, e86372. doi:10.1371/journal. pone. 0086372

Guiney, S. J., Adlard, P. A., Bush, A. I. et al. (2017). Ferroptosis and cell death mechanisms in Parkinson's disease. Neurochem Int 104, 34-48. doi:10.1016/j.neuint.2017.01.004

Gutbier, S., May, P., Berthelot, S. et al. (2018a). Major changes of cell function and toxicant sensitivity in cultured cells undergoing mild, quasi-natural genetic drift. Arch Toxicol 92, 34873503. doi:10.1007/s00204-018-2326-5

Gutbier, S., Spreng, A. S., Delp, J. et al. (2018b). Prevention of neuronal apoptosis by astrocytes through thiol-mediated stress response modulation and accelerated recovery from proteotoxic stress. Cell Death Differ 25, 2101-2117. doi:10.1038/ s41418-018-0229-x

Haenseler, W., Sansom, S. N., Buchrieser, J. et al. (2017). A highly efficient human pluripotent stem cell microglia model displays a neuronal-co-culture-specific expression profile and inflammatory response. Stem Cell Rep 8, 1727-1742. doi:10.1016/j.stemcr.2017.05.017

Hama, H., Hioki, H., Namiki, K. et al. (2015). ScaleS: An optical clearing palette for biological imaging. Nat Neurosci 18, 15181529. doi:10.1038/nn.4107

Hansson, O., Castilho, R. F., Kaminski Schierle, G. S. et al. (2000). Additive effects of caspase inhibitor and lazaroid on the survival of transplanted rat and human embryonic dopamine neurons. Exp Neurol 164, 102-111. doi:10.1006/exnr. 2000.7406

Harris, G., Hogberg, H., Hartung, T. et al. (2017). 3D differentiation of LUHMES cell line to study recovery and delayed neurotoxic effects. Curr Protoc Toxicol 73, 11.23.11-11.23.28. doi:10.1002/cptx.29

Harris, G., Eschment, M., Orozco, S. P. et al. (2018). Toxicity, recovery, and resilience in a $3 \mathrm{D}$ dopaminergic neuronal in vitro model exposed to rotenone. Arch Toxicol 92, 2587-2606. doi:10.1007/s00204-018-2250-8
Hartung, T. and Leist, M. (2008). Food for thought ... on the evolution of toxicology and the phasing out of animal testing. ALTEX 25, 91-102. doi:10.14573/altex.2008.2.91

Hirt, U. A., Gantner, F. and Leist, M. (2000). Phagocytosis of nonapoptotic cells dying by caspase-independent mechanisms. J Immunol 164, 6520-6529. doi:10.4049/jimmunol. 164.12.6520

Honegger, P. and Richelson, E. (1977). Kainic acid alters neurochemical development in fetal rat brain aggregating cell cultures. Brain Res 138, 580-584. doi:10.1016/0006-8993 (77)90698-9

Honegger, P. and Richelson, E. (1979). Neurotransmitter synthesis, storage and release by aggregating cell cultures of rat brain. Brain Res 162, 89-101. doi:10.1016/0006-8993(79)90758-3

Howes, A. L., Richardson, R. D., Finlay, D. et al. (2014). 3-dimensional culture systems for anti-cancer compound profiling and high-throughput screening reveal increases in EGFR inhibitor-mediated cytotoxicity compared to monolayer culture systems. PLoS One 9, e108283. doi:10.1371/journal. pone. 0108283

Jay, T. R., von Saucken, V. E., Munoz, B. et al. (2019). TREM2 is required for microglial instruction of astrocytic synaptic engulfment in neurodevelopment. Glia 67, 1873-1892. doi:10.1002/glia.23664

Ji, R. R., Donnelly, C. R. and Nedergaard, M. (2019). Astrocytes in chronic pain and itch. Nat Rev Neurosci 20, 667-685. doi:10.1038/s41583-019-0218-1

Jo, J., Xiao, Y., Sun, A. X. et al. (2016). Midbrain-like organoids from human pluripotent stem cells contain functional dopaminergic and neuromelanin-producing neurons. Cell Stem Cell 19, 248-257. doi:10.1016/j.stem.2016.07.005

Jones, E. V., Cook, D. and Murai, K. K. (2012). A neuron-astrocyte co-culture system to investigate astrocyte-secreted factors in mouse neuronal development. Methods Mol Biol 814, 341352. doi:10.1007/978-1-61779-452-0_22

Kadoshima, T., Sakaguchi, H., Nakano, T. et al. (2013). Self-organization of axial polarity, inside-out layer pattern, and species-specific progenitor dynamics in human ES cell-derived neocortex. Proc Natl Acad Sci U S A 110, 20284-20289. doi:10.1073/pnas.1315710110

Kelava, I. and Lancaster, M. A. (2016). Stem cell models of human brain development. Cell Stem Cell 18, 736-748. doi:10.1016/j.stem.2016.05.022

Kim, C. and Park, S. (2018). IGF-1 protects SH-SY5Y cells against $\mathrm{MPP}^{+}$-induced apoptosis via $\mathrm{PI} 3 \mathrm{~K} / \mathrm{PDK}-1 /$ Akt pathway. Endocr Connect 7, 443-455. doi:10.1530/EC-17-0350

Kim, S., Lowe, A., Dharmat, R. et al. (2019). Generation, transcriptome profiling, and functional validation of cone-rich human retinal organoids. Proc Natl Acad Sci U S A 116, 1082410833. doi:10.1073/pnas.1901572116

Klapper, S. D., Garg, P., Dagar, S. et al. (2019). Astrocyte lineage cells are essential for functional neuronal differentiation and synapse maturation in human iPSC-derived neural networks. Glia 67, 1893-1909. doi:10.1002/glia.23666

Krencik, R., Seo, K., van Asperen, J. V. et al. (2017). Systematic three-dimensional coculture rapidly recapitulates interac- 
tions between human neurons and astrocytes. Stem Cell Rep 9, 1745-1753. doi:10.1016/j.stemcr.2017.10.026

Krug, A. K., Balmer, N. V., Matt, F. et al. (2013). Evaluation of a human neurite growth assay as specific screen for developmental neurotoxicants. Arch Toxicol 87, 2215-2231. doi:10.1007/ s00204-013-1072-y

Krug, A. K., Gutbier, S., Zhao, L. et al. (2014). Transcriptional and metabolic adaptation of human neurons to the mitochondrial toxicant $\mathrm{MPP}^{+}$. Cell Death Dis 5, e1222. doi:10.1038/ cddis.2014.166

Lancaster, M. A., Renner, M., Martin, C. A. et al. (2013). Cerebral organoids model human brain development and microcephaly. Nature 501, 373-379. doi:10.1038/nature12517

Lancaster, M. A. and Knoblich, J. A. (2014). Generation of cerebral organoids from human pluripotent stem cells. Nat Protoc 9, 2329-2340. doi:10.1038/nprot.2014.158

Lancaster, M. A., Corsini, N. S., Wolfinger, S. et al. (2017). Guided self-organization and cortical plate formation in human brain organoids. Nat Biotechnol 35, 659-666. doi:10.1038/nbt.3906

Leist, M., Hartung, T. and Nicotera, P. (2008). The dawning of a new age of toxicology. ALTEX 25, 103-114. doi:10.14573/ altex.2008.2.103

Leist, M. and Hartung, T. (2013). Inflammatory findings on species extrapolations: Humans are definitely no 70-kg mice. Arch Toxicol 87, 563-567. doi:10.1007/s00204-013-1038-0

Li, K., Li, J., Zheng, J. et al. (2019). Reactive astrocytes in neurodegenerative diseases. Aging Dis 10, 664-675. doi:10.14336/ AD.2018.0720

Livni, L., Lees, J. G., Barkl-Luke, M. E. et al. (2019). Dorsal root ganglion explants derived from chemotherapy-treated mice have reduced neurite outgrowth in culture. Neurosci Lett 694, 14-19. doi:10.1016/j.neulet.2018.11.016

Lotharius, J., Falsig, J., van Beek, J. et al. (2005). Progressive degeneration of human mesencephalic neuron-derived cells triggered by dopamine-dependent oxidative stress is dependent on the mixed-lineage kinase pathway. J Neurosci 25, 6329. doi:10.1523/JNEUROSCI.1746-05.2005

Manders, E., Verbeek, F. and Aten, J. (1993). Measurement of co-localization of objects in dual-colour confocal images. J Microsc 169, 375-382. doi:10.1111/j.1365-2818.1993. tb03313.x

Marton, R. M. and Pasca, S. P. (2016). Neural differentiation in the third dimension: Generating a human midbrain. Cell Stem Cell 19, 145-146. doi:10.1016/j.stem.2016.07.017

Marx, U., Andersson, T. B., Bahinski, A. et al. (2016). Biology-inspired microphysiological system approaches to solve the prediction dilemma of substance testing. ALTEX 33, 272321. doi:10.14573/altex.1603161

Monzel, A. S., Smits, L. M., Hemmer, K. et al. (2017). Derivation of human midbrain-specific organoids from neuroepithelial stem cells. Stem Cell Rep 8, 1144-1154. doi:10.1016/j.stemcr. 2017.03.010

Muguruma, K., Nishiyama, A., Kawakami, H. et al. (2015). Self-organization of polarized cerebellar tissue in 3D culture of human pluripotent stem cells. Cell Rep 10, 537-550. doi:10.1016/j.celrep.2014.12.051
Nedergaard, M., Ransom, B. and Goldman, S. A. (2003). New roles for astrocytes: Redefining the functional architecture of the brain. Trends Neurosci 26, 523-530. doi:10.1016/j.tins.2003.08.008

Nzou, G., Wicks, R. T., Wicks, E. E. et al. (2018). Human cortex spheroid with a functional blood brain barrier for high-throughput neurotoxicity screening and disease modeling. Sci Rep 8, 7413. doi:10.1038/s41598-018-25603-5

Ormel, P. R., Vieira de Sá, R., van Bodegraven, E. J. et al. (2018). Microglia innately develop within cerebral organoids. Nat Comm 9, 4167. doi:10.1038/s41467-018-06684-2

Palm, T., Bolognin, S., Meiser, J. et al. (2015). Rapid and robust generation of long-term self-renewing human neural stem cells with the ability to generate mature astroglia. Sci Rep 5, 16321. doi:10.1038/srep16321

Pamies, D. and Hartung, T. (2017). 21 $1^{\text {st }}$ century cell culture for $21^{\text {st }}$ century toxicology. Chem Res Toxicol 30, 43-52. doi:10.1021/acs.chemrestox.6b00269

Pamies, D., Barreras, P., Block, K. et al. (2017). A human brain microphysiological system derived from induced pluripotent stem cells to study neurological diseases and toxicity. ALTEX 34, 362-376. doi:10.14573/altex.1609122

Pampaloni, F., Reynaud, E. G. and Stelzer, E. H. K. (2007). The third dimension bridges the gap between cell culture and live tissue. Nat Rev Mol Cell Biol 8, 839. doi:10.1038/nrm2236

Pasca, A. M., Sloan, S. A., Clarke, L. E. et al. (2015). Functional cortical neurons and astrocytes from human pluripotent stem cells in 3D culture. Nat Methods 12, 671-678. doi:10.1038/ nmeth. 3415

Pizzurro, D. M., Dao, K. and Costa, L. G. (2014). Astrocytes protect against diazinon- and diazoxon-induced inhibition of neurite outgrowth by regulating neuronal glutathione. Toxicology 318, 59-68. doi:10.1016/j.tox.2014.01.010

Polioudakis, D., de la Torre-Ubieta, L., Langerman, J. et al. (2019). A single-cell transcriptomic atlas of human neocortical development during mid-gestation. Neuron 103, 785-801.e8. doi:10.1016/j.neuron.2019.06.011

Qian, X., Nguyen, H. N., Song, M. M. et al. (2016). Brain-region-specific organoids using mini-bioreactors for modeling ZIKV exposure. Cell 165, 1238-1254. doi:10.1016/j. cell.2016.04.032

Quadrato, G., Nguyen, T., Macosko, E. Z. et al. (2017). Cell diversity and network dynamics in photosensitive human brain organoids. Nature 545, 48-53. doi:10.1038/nature22047

Rathinam, M. L., Watts, L. T., Narasimhan, M. et al. (2012). Astrocyte mediated protection of fetal cerebral cortical neurons from rotenone and paraquat. Environ Toxicol Pharmacol 33, 353-360. doi:10.1016/j.etap.2011.12.027

Ravi, M., Paramesh, V., Kaviya, S. R. et al. (2015). 3D cell culture systems: Advantages and applications. J Cell Physiol 230, 16-26. doi:10.1002/jcp. 24683

Riccardi, C. and Nicoletti, I. (2006). Analysis of apoptosis by propidium iodide staining and flow cytometry. Nat Protoc 1, 1458-1461. doi:10.1038/nprot.2006.238

Roque, P. J. and Costa, L. G. (2017). Co-culture of neurons and microglia. Curr Protoc Toxicol 74, 11.24.11-11.24.17. doi:10.1002/cptx.32 
Schildknecht, S., Poltl, D., Nagel, D. M. et al. (2009). Requirement of a dopaminergic neuronal phenotype for toxicity of low concentrations of 1-methyl-4-phenylpyridinium to human cells. Toxicol Appl Pharmacol 241, 23-35. doi:10.1016/j.taap. 2009.07.027

Schildknecht, S., Pape, R., Muller, N. et al. (2011). Neuroprotection by minocycline caused by direct and specific scavenging of peroxynitrite. J Biol Chem 286, 4991-5002. doi:10.1074/ jbc.M110.169565

Schildknecht, S., Gerding, H. R., Karreman, C. et al. (2013a). Oxidative and nitrative alpha-synuclein modifications and proteostatic stress: Implications for disease mechanisms and interventions in synucleinopathies. J Neurochem 125, 491-511. doi:10.1111/jnc. 12226

Schildknecht, S., Karreman, C., Poltl, D. et al. (2013b). Generation of genetically-modified human differentiated cells for toxicological tests and the study of neurodegenerative diseases. ALTEX 30, 427-444. doi:10.14573/altex.2013.4.427

Schildknecht, S., Pape, R., Meiser, J. et al. (2015). Preferential extracellular generation of the active parkinsonian toxin $\mathrm{MPP}^{+}$by transporter-independent export of the intermediate $\mathrm{MPP}^{+}$. Antioxid Redox Signal 23, 1001-1016. doi:10.1089/ ars.2015.6297

Schmidt, B. Z., Lehmann, M., Gutbier, S. et al. (2017). In vitro acute and developmental neurotoxicity screening: An overview of cellular platforms and high-throughput technical possibilities. Arch Toxicol 91, 1-33. doi:10.1007/s00204-0161805-9

Scholz, D., Poltl, D., Genewsky, A. et al. (2011). Rapid, complete and large-scale generation of post-mitotic neurons from the human LUHMES cell line. J Neurochem 119, 957-971. doi:10.1111/j.1471-4159.2011.07255.x

Scholz, D., Chernyshova, Y., Uckert, A. K. et al. (2018). Reduced Abeta secretion by human neurons under conditions of strongly increased BACE activity. J Neurochem 147, 256-274. doi:10.1111/jnc. 14467

Semple, B. D., Blomgren, K., Gimlin, K. et al. (2013). Brain development in rodents and humans: Identifying benchmarks of maturation and vulnerability to injury across species. Prog Neurobiol 106-107, 1-16. doi:10.1016/j.pneurobio.2013.04.001

Serra, M., Leite, S. B., Brito, C. et al. (2007). Novel culture strategy for human stem cell proliferation and neuronal differentiation. J Neurosci Res 85, 3557-3566. doi:10.1002/jnr.21451

Shi, Y., Inoue, H., Wu, J. C. et al. (2017). Induced pluripotent stem cell technology: A decade of progress. Nat Rev Drug Discov 16, 115-130. doi:10.1038/nrd.2016.245

Sidoryk-Wegrzynowicz, M., Wegrzynowicz, M., Lee, E. et al. (2011). Role of astrocytes in brain function and disease. Toxicol Pathol 39, 115-123. doi:10.1177/0192623310385254

Simão, D., Terrasso, A. P., Teixeira, A. P. et al. (2016). Functional metabolic interactions of human neuron-astrocyte $3 \mathrm{D}$ in vitro networks. Sci Rep 6, 33285. doi:10.1038/srep33285

Singh, N., Lawana, V., Luo, J. et al. (2018). Organophosphate pesticide chlorpyrifos impairs STAT1 signaling to induce dopaminergic neurotoxicity: Implications for mitochondria me- diated oxidative stress signaling events. Neurobiol Dis 117, 82-113. doi:10.1016/j.nbd.2018.05.019

Sipes, N. S., Martin, M. T., Kothiya, P. et al. (2013). Profiling 976 ToxCast chemicals across 331 enzymatic and receptor signaling assays. Chem Res Toxicol 26, 878-895. doi:10.1021/ tx400021f

Skirzewski, M., Karavanova, I., Shamir, A. et al. (2018). ErbB4 signaling in dopaminergic axonal projections increases extracellular dopamine levels and regulates spatial/working memory behaviors. Mol Psychiatry 23, 2227-2237. doi:10.1038/ mp.2017.132

Sloan, S. A., Darmanis, S., Huber, N. et al. (2017). Human astrocyte maturation captured in 3D cerebral cortical spheroids derived from pluripotent stem cells. Neuron 95, 779-790.e6. doi:10.1016/j.neuron.2017.07.035

Smirnova, L., Harris, G., Delp, J. et al. (2016). A LUHMES 3D dopaminergic neuronal model for neurotoxicity testing allowing long-term exposure and cellular resilience analysis. Arch Toxicol 90, 2725-2743. doi:10.1007/s00204-015-1637-z

Smits, L. M., Reinhardt, L., Reinhardt, P. et al. (2019). Modeling Parkinson's disease in midbrain-like organoids. NPJ Parkinsons Dis 5, 5. doi:10.1038/s41531-019-0078-4

Stary, C. M., Sun, X. and Giffard, R. G. (2015). Astrocytes protect against isoflurane neurotoxicity by buffering pro-brainderived neurotrophic factor. Anesthesiology 123, 810-819. doi:10.1097/ALN.0000000000000824

Stiegler, N. V., Krug, A. K., Matt, F. et al. (2011). Assessment of chemical-induced impairment of human neurite outgrowth by multiparametric live cell imaging in high-density cultures. Toxicol Sci 121, 73-87. doi:10.1093/toxsci/kfr034

Sun, F., Anantharam, V., Zhang, D. et al. (2006). Proteasome inhibitor MG-132 induces dopaminergic degeneration in cell culture and animal models. Neurotoxicology 27, 807-815. doi:10.1016/j.neuro.2006.06.006

Takahashi, K., Tanabe, K., Ohnuki, M. et al. (2007). Induction of pluripotent stem cells from adult human fibroblasts by defined factors. Cell 131, 861-872. doi:10.1016/j.cell.2007.11.019

Thomson, J. A., Itskovitz-Eldor, J., Shapiro, S. S. et al. (1998). Embryonic stem cell lines derived from human blastocysts. Science 282, 1145-1147. doi:10.1126/science.282.5391.1145

Tong, Z. B., Hogberg, H., Kuo, D. et al. (2017). Characterization of three human cell line models for high-throughput neuronal cytotoxicity screening. J Appl Toxicol 37, 167-180. doi:10.1002/jat.3334

Trujillo, C. A., Gao, R., Negraes, P. D. et al. (2019). Complex oscillatory waves emerging from cortical organoids model early human brain network development. Cell Stem Cell 25, 558569.e557. doi:10.1016/j.stem.2019.08.002

Tukker, A. M., Wijnolts, F. M. J., de Groot, A. et al. (2018). Human iPSC-derived neuronal models for in vitro neurotoxicity assessment. Neurotoxicology 67, 215-225. doi:10.1016/j.neuro. 2018.06.007

van der Worp, H. B., Howells, D. W., Sena, E. S. et al. (2010). Can animal models of disease reliably inform human studies? PLoS Med 7, e1000245. doi:10.1371/journal.pmed.1000245 van Wilgenburg, B., Browne, C., Vowles, J. et al. (2013). Effi- 
cient, long term production of monocyte-derived macrophages from human pluripotent stem cells under partly-defined and fully-defined conditions. PLoS One 8, e71098. doi:10.1371/ journal.pone.0071098

Verkhratsky, A. and Nedergaard, M. (2018). Physiology of astroglia. Physiol Rev 98, 239-389. doi:10.1152/physrev.00042. 2016

Wang, X. F. and Cynader, M. S. (2000). Astrocytes provide cysteine to neurons by releasing glutathione. J Neurochem 74, 1434-1442. doi:10.1046/j.1471-4159.2000.0741434.x

Wang, Y., Wang, L., Zhu, Y. et al. (2018). Human brain organoidon-a-chip to model prenatal nicotine exposure. Lab Chip 18, 851-860. doi:10.1039/C7LC01084B

Wilton, D. K., Dissing-Olesen, L. and Stevens, B. (2019). Neuron-glia signaling in synapse elimination. Annu Rev Neurosci 42, 107-127. doi:10.1146/annurev-neuro-070918-050306

Witt, B., Meyer, S., Ebert, F. et al. (2017). Toxicity of two classes of arsenolipids and their water-soluble metabolites in human differentiated neurons. Arch Toxicol 91, 3121-3134. doi:10.1007/s00204-017-1933-x

Wolpaw, A. J., Shimada, K., Skouta, R. et al. (2011). Modulatory profiling identifies mechanisms of small molecule-induced cell death. Proc Natl Acad Sci U S A 108, E771-E780. doi:10.1073/pnas.1106149108

Yakoub, A. M. (2019). Cerebral organoids exhibit mature neurons and astrocytes and recapitulate electrophysiological activity of the human brain. Neural Regen Res 14, 757-761. doi:10.4103/1673-5374.249283

Yoon, S. J., Elahi, L. S., Pasca, A. M. et al. (2019). Reliability of human cortical organoid generation. Nat Methods 16, 75-78. doi:10.1038/s41592-018-0255-0
Zhang, X. M., Yin, M. and Zhang, M. H. (2014). Cell-based assays for Parkinson's disease using differentiated human LUHMES cells. Acta Pharmacol Sin 35, 945-956. doi:10.1038/aps.2014.36

Zhang, Y., Sloan, S. A., Clarke, L. E. et al. (2016). Purification and characterization of progenitor and mature human astrocytes reveals transcriptional and functional differences with mouse. Neuron 89, 37-53. doi:10.1016/j.neuron.2015.11.013

Zimmer, B., Kuegler, P. B., Baudis, B. et al. (2011). Coordinated waves of gene expression during neuronal differentiation of embryonic stem cells as basis for novel approaches to developmental neurotoxicity testing. Cell Death Differ 18, 383-395. doi:10.1038/cdd.2010.109

\section{Conflict of interest}

The authors declare no conflict of interest.

\section{Acknowledgements}

This work was supported by the BMBF, EFSA, the DK-EPA (MST-667-00205), and the DFG (Konstanz Research School of Chemical Biology; KoRS-CB). It has received funding from the European Union's Horizon 2020 research and innovation programme under grant agreements No. 681002 (EU-ToxRisk) and No. 825759 (ENDpoiNTs). We kindly thank Ncardia, Germany, for providing the Astro.4U cells. We further thank Luxendo, Germany, for providing a MuVi SPIM microscope and their experimental support. We are grateful to M. Kapitza, H. Leisner, P. Lutzenburger, M. Prokofjew, L. Maschek and the staff of the bioimaging center (BIC) for invaluable experimental support. 